\title{
Cosmic dance in the Shapley Concentration Core
}

\section{A study of the radio emission of the BCGs and tailed radio galaxies} \author{
S. Bardelli ${ }^{5}$, F. Gastaldello ${ }^{8}$, A. Grado ${ }^{7}$, C. P. Haines ${ }^{9}$, L. Limatola $^{7}$, and M. Rossetti ${ }^{8}$ \\ 1 INAF - Istituto di Radioastronomia, Via Gobetti 101, 40129 Bologna, Italy \\ e-mail: gabriella.di.gennaro@cfa.harvard.edu \\ 2 Harvard-Smithsonian Center for Astrophysics, 60 Garden Street, Cambridge, MA 02138, USA \\ 3 Leiden Observatory, Leiden University, PO Box 9513, 2300 RA Leiden, The Netherlands \\ 4 Dipartimento di Fisica e Astronomia, Università di Bologna, Via Gobetti 93/2, 40129 Bologna, Italy \\ 5 INAF-Astrophysics and Space Science Observatory Bologna, Via Gobetti 93/3, 40129 Bologna, Italy \\ ${ }^{6}$ Naval Research Laboratory, 4555 Overlook Avenue SW, Code 7213, Washington, DC 20375, USA \\ 7 INAF - Osservatorio Astronomico di Capodimonte, Salita Moiariello 16, 80131 Napoli, Italy \\ 8 INAF - IASF Milano, Via Bassini 15, 20133 Milano, Italy \\ 9 INAF - Osservatorio Astronomico di Brera, Via Brera 28, 20121 Milano, Italy
}

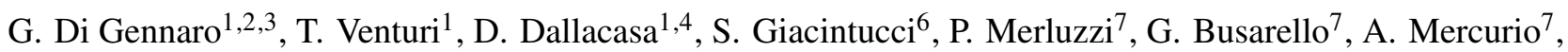

Received 8 February 2018 / Accepted 11 July 2018

\begin{abstract}
Context. The Shapley Concentration $(\langle z\rangle \approx 0.048)$ covers several degrees in the southern hemisphere, and includes galaxy clusters in advanced evolutionary stages, groups of clusters in the very early stages of merger, fairly massive clusters with ongoing accretion activity, and smaller groups located in filaments in the regions between the main clusters.

Aims. With the goal to investigate the role of cluster mergers and accretion on the radio galaxy population, we performed a multiwavelength study of the brightest cluster galaxies (BCGs) and of the galaxies showing extended radio emission in the cluster complexes of Abell 3528 and Abell 3558. In total, our study is based on a sample of 12 galaxies.

Methods. We observed the clusters with the Giant Metrewave Radio Telescope (GMRT) at 235, 325, and 610 MHz, and with the Very Large Array (VLA) at $8.46 \mathrm{GHz}$. We complemented our study with the TIFR GMRT Sky Survey (TGSS) at $150 \mathrm{MHz}$, the Sydney University Molonglo Sky Survey (SUMSS) at $843 \mathrm{MHz}$, and the Australia Telescope Compact array (ATCA) at 1380, 1400, 2380, and $4790 \mathrm{MHz}$ data. Finally, optical imaging with the VLT Survey Telescope (VST) is also available for the host galaxies as well as the mid-infrared coverage with the Wide-Field Infrared Survey Explorer (WISE).

Results. We found significant differences in the properties of the radio emission of the BCGs in the two cluster complexes. The BCGs in the A 3528 complex and in A 3556, which are relaxed cool-core objects, are powerful active radio galaxies. They also present hints of restarted activity. On the contrary, the BCGs in A 3558 and A 3562, which are well-known merging systems, are very faint, or quiet, in the radio band. The optical and infrared properties of the galaxies, on the other hand, are fairly similar in the two complexes, showing all passive red galaxies.

Conclusions. Our study shows remarkable differences in the radio properties of the BGCs, which we relate to the different dynamical state of the host cluster. On the contrary, the lack of changes between such different environments in the optical band suggests that the dynamical state of galaxy clusters does not affect the optical counterparts of the radio galaxies, at least over the lifetime of the radio emission.
\end{abstract}

Key words. radiation mechanisms: non-thermal - galaxies: clusters: general - galaxies: elliptical and lenticular, cD radio continuum: galaxies

\section{Introduction}

Cluster mergers are the most energetic phenomena in the Universe, releasing energies of the order of $10^{63-64} \mathrm{erg}$ depending on the mass of the clusters and on the relative velocity of the merging halos. In a hierarchical cold dark matter scenario, these phenomena are the natural way to form rich clusters of galaxies, but it is still unknown how such amounts of gravitational energy are dissipated and what are the consequences on the environment (intracluster medium, magnetic fields and in-situ relativistic particles) and on the galaxy population.

Mergers and group accretion leave important footprints in the radio band. Beyond the well-known diffuse cluster sources in the form of halos, relics and mini-halos, whose relation to the cluster dynamical state is an established result from both the observational and theoretical point of view (e.g. Markevitch \& Vikhlinin 2007; Venturi et al. 2008; van Weeren et al. 2011; ZuHone et al. 2013; Cassano et al. 2013; Brunetti \& Jones 2014; Giacintucci et al. 2014a, 2017), further crucial information on the cluster dynamics and on the interaction between the radio plasma and the intracluster medium (ICM) comes from the galaxy population. Double radio galaxies in galaxy clusters (mainly Fanaroff-Riley type I, or transition objects I/II, i.e FRI and FRI/II, respectively; see Fanaroff \& Riley 1974) often show bent and distorted jets and lobes, which are usually classified on the basis of the angle between the two jets (Miley 1980). Head-tailed 
(HT) and narrow-angle tailed (NAT) radio galaxies (sometimes also called C-shaped sources) are associated with nondominant cluster galaxies, and are interpreted as the result of ram pressure exerted by the intergalactic medium on the double-sided radio emission (O'Dea \& Owen 1985). Wide-angle tailed (WAT) radio galaxies, on the other hand are often associated with the central brightest cluster galaxies (BCGs) and their shape is explained as a combination of bulk motion of the ICM as a consequence of clusters mergers and "cluster weather" (Burns 1998 and see Feretti \& Venturi 2002, for a review).

BCGs are the most massive and luminous elliptical galaxies in the Universe. They are located in the proximity of the bottom of the cluster potential well, and are usually active in the radio band: the fraction of radio loud BCGs is much higher than for the other elliptical galaxies, reaching up to $30 \%$ for $L_{1.4 \mathrm{GHz}}>10^{23} \mathrm{~W} \mathrm{~Hz}^{-1}$, and the normalisation of their radio luminosity function is the highest among elliptical galaxies (Best et al. 2007). Moreover, the radio properties of these radio galaxies are strongly dependent on the central properties of the host cluster, both in the local Universe and at intermediate redshift. The probability of finding a radio-loud BCG is much higher in relaxed clusters, and such a probability increases if we are looking at "strong" cool-core clusters (Cavagnolo et al. 2008; Sun 2009; Kale et al. 2015). The active galactic nuclei (AGN) feedback of the BCGs is also an established result: the nuclear radio emission may take the form of mechanical feedback to the ICM, preventing gas cooling in dynamically relaxed environments (see McNamara \& Nulsen 2007, 2012).

The galaxy environment is a determinant for both the morphology-density (Dressler 1980; Dressler et al. 1997) and the star-formation-density (Butcher \& Oemler 1984; Lewis et al. 2002; Kauffmann et al. 2004) relations observed at redshift $z \sim 0$. These relations show that late-type, blue, star-forming galaxies are predominant in the field, while early-type, red, passive galaxies are preferentially found in galaxy clusters. This suggests that blue galaxies accreted from the field in the past have been transformed into the passive lenticular and dwarf ellipticals found in local clusters. The proposed and investigated mechanisms affecting the galaxy properties include gravitational and tidal interactions amongst galaxies (Toomre \& Toomre 1972; Moore et al. 1996), between galaxies and the cluster gravitational field (Byrd \& Valtonen 1990), galaxy mergers (Barnes \& Hernquist 1991), group-cluster collisions (Bekki 2001), ram-pressure stripping (Gunn \& Gott 1972), viscous stripping (Nulsen 1982), evaporation (Cowie \& Songalia 1977), and "starvation" (Larson et al. 1980). Although the effects, timescales and efficiencies of these physical processes can be different, all together they serve to transform galaxies by disturbing their kinematics, depleting their gas reservoir and so ultimately quenching their star formation which can even be enhanced in the first phase of the transformation. Galaxies that pass through the cluster centre are those mostly affected by such mechanisms, while those just falling into the cluster outskirts or moving on a tangential orbit may retain part of their gas. The impact of the large-scale environment, such as the highest overdensities (i.e. galaxy clusters and the so-called superclusters), on star formation and other galaxy properties is, however, still unclear. Superclusters provide an excellent chance to study such effects. In these dynamically active and locally dense structures processes such as cluster-cluster collisions and mergers in different phases together with a wide and inter-connected range of environments (from cluster cores to filaments) dramati- cally enhance the probability of observing evidence of environmental effects on galaxy evolution at the same epoch (e.g. Merluzzi et al. 2015).

In this paper, we address the role of cluster mergers and group accretion in shaping the radio and optical properties of galaxies in the core region of the Shapley Concentration, where many clusters and groups in different evolutionary stages are located. In particular, we present a detailed radio-optical study of the BCGs and tailed radio galaxies of the A 3558 and A 3528 cluster complexes based on data taken with the Giant Metrewave Radio Telescope (GMRT) and the Very Large Array (VLA), combined with the literature and archival information from the TIFR GMRT Sky Survey (TGSS), the Sydney University Molonglo Sky Survey (SUMSS), the VLA, and the Australia Telescope Compact Array (ATCA). The global radio analysis spans almost two orders of magnitude in frequency, from $150 \mathrm{MHz}$ to $8.46 \mathrm{GHz}$. Our analysis is complemented by the optical and near-infrared (near-IR) information from the Shapley Supercluster Survey (ShaSS; Merluzzi et al. 2015) together with the IR coverage with the Wide-Field Infrared Survey Explorer (WISE).

The paper is organised as follows: in Sect. 2 we provide an overview of the region of the Shapley Concentration under investigation; in Sect. 3 we describe the radio and optical observations and detail the radio analysis; the radio images and the galaxies sample are presented in Sects. 4 and 5. The radio spectral study follows in Sect. 6. In Sect. 7 we present the results of the optical analysis. Finally, our results and conclusions are discussed and interpreted in Sects. 8 and 9.

Throughout the paper we use the convention $S_{v} \propto v^{-\alpha}$. We assume a standard cosmology with $H_{0}=70 \mathrm{~km} \mathrm{~s}^{-1} \mathrm{Mpc}-1$, $\Omega_{\mathrm{m}}=0.3$ and $\Omega_{\Lambda}=0.7$ is assumed, which implies a conversion factor of $0.928 \mathrm{kpc} \operatorname{arcsec}^{-1}$ and an average luminosity distance $210 \mathrm{Mpc}$.

\section{The Shapley Concentration in context}

The Shapley Concentration (Shapley 1930) is the richest and most massive concentration of galaxy clusters, or supercluster, in the local Universe (e.g. Raychaudhury 1989; Scaramella et al. 1989; Vettolani et al. 1990; Zucca et al. 1993). It is located in the southern sky and lies behind the Hydra-Centaurus cluster. Overall, the structure covers a redshift range $0.033 \lesssim z \lesssim 0.06$ (Quintana et al. 1995, 1997), with a mean redshift $z \approx 0.048$. Due to the very high overdensity of galaxy clusters, the innermost region of the Shapley Concentration is dynamically active, and thanks to its proximity it is an ideal place to investigate the effects of group accretion and cluster mergers. The masses and the bolometric luminosities of the individual clusters and groups range between $M_{500} \approx 0.1-6 \times 10^{14} \mathrm{~h}^{-1} M_{\odot}^{1}$ (Reisenegger et al. 2000) and $L_{X} \approx 0.5-6.7 \times 10^{44} \mathrm{erg} \mathrm{s}^{-1}$ (de Filippis et al. 2005), respectively.

The A 3558 cluster complex is considered the centre of the Shapley Concentration Core and consists of a chain of three Abell clusters (A 3556, A 3558 and A 3562) and two smaller groups (SC 1327-312 and SC 1329-313). It extends for about $7.5 \mathrm{~h}^{-1} \mathrm{Mpc}$ in the east-west direction, at an average redshift of $\langle z\rangle \approx 0.048$. Studies in the optical (Bardelli et al. 1998a,b; Merluzzi et al. 2015), X-ray (Markevitch \& Vikhlinin 1997; Ettori et al. 2000; Rossetti et al. 2007; Ghizzardi et al. 2010), and radio (Venturi et al. 2000, 2003, 2017; Giacintucci et al. 2004, 2005) bands provide several pieces of evidence in support

\footnotetext{
$M_{500}$ is defined as the mass calculated at the radius where the cluster density exceeds 500 times the critical density of the Universe.
} 
Table 1. Logs of the observations.

\begin{tabular}{|c|c|c|c|c|c|c|c|}
\hline Cluster & RA, Dec (J2000) & Array & Project & Observing date & $\begin{array}{c}v \\
(\mathrm{MHz})\end{array}$ & $\begin{array}{c}\Delta v \\
(\mathrm{MHz})\end{array}$ & $\begin{array}{c}\text { Total time } \\
\text { on source }(\mathrm{h})\end{array}$ \\
\hline \multirow[t]{4}{*}{ A $3528 \mathrm{~N}$} & $125420,-290230$ & GMRT & 05TVa01 & 5/6-Apr.-2004 & 235 & 8 & $4^{a}$ \\
\hline & $125420,-290230$ & GMRT & $05 \mathrm{TVa} 01$ & 5/6-Apr.-2004 & 610 & 16 & $4^{a}$ \\
\hline & $125422,-290102$ & VLA-CnB & AV246 & 13-Mar.-2000 & 8460 & 100 & $0.25^{b}$ \\
\hline & $125421,-290416$ & VLA-CnB & AV246 & 13-Mar.-2000 & 8460 & 100 & $0.25^{b}$ \\
\hline \multirow[t]{3}{*}{ A $3528 \mathrm{~S}$} & $125500,-294000$ & GMRT & $05 \mathrm{TVa} 01$ & 5/6-Apr.-2004 & 235 & 8 & $4^{a}$ \\
\hline & $125500,-294000$ & GMRT & $05 \mathrm{TVa} 01$ & 5/6-Apr.-2004 & 610 & 16 & $4^{a}$ \\
\hline & $125451,-291620$ & VLA-CnB & AV246 & 3-Mar.-2000 & 8460 & 100 & $0.25^{b}$ \\
\hline \multirow[t]{2}{*}{ A 3532} & $125630,-303000$ & GMRT & $05 \mathrm{TVa} 01$ & 5/6-Apr.-2004 & 235 & 8 & $4^{a}$ \\
\hline & $125630,-303000$ & GMRT & $05 \mathrm{TVa} 01$ & 5/6-Apr.-2004 & 610 & 16 & $4^{a}$ \\
\hline \multirow{2}{*}{ A 3556} & $132400,-313800$ & GMRT & $05 \mathrm{TVa} 01$ & 5/6-Apr.-2004 & 235 & 8 & $4^{a}$ \\
\hline & $132400,-313800$ & GMRT & $05 \mathrm{TVa} 01$ & 5/6-Apr.-2004 & 610 & 16 & $4^{a}$ \\
\hline \multirow[t]{2}{*}{ A 3558} & $132754,-312932$ & GMRT & 22_039 & 30-Aug.-2012 & 325 & 32 & $8^{c}$ \\
\hline & $132754,-312932$ & GMRT & 22_039 & 02-May-2015 & 610 & 32 & $7^{c}$ \\
\hline
\end{tabular}

Notes. ${ }^{(a)}$ Observations of project $05 \mathrm{TVa} 01$ were carried out on two consecutive days with the dual $235 / 610 \mathrm{MHz}$ receiver, for a total of $8 \mathrm{~h}$ each day. We cycled among the four pointing centres every $20 \mathrm{~min} .{ }^{(b)}$ The total duration of project AV246 was one hour, and the observations were carried out switching among the three pointing centres every $10 \mathrm{~min} .{ }^{(c)}$ Full track observations.

of the idea that the whole region between A 3558 and A 3562 is unrelaxed.

The A 3528 cluster complex is located at a projected distance of approximately $19 \mathrm{~h}^{-1} \mathrm{Mpc}$ north-west of A 3558, and it is formed by the three Abell clusters A 3528, A 3530, and A 3532. It extends for about $7.5 \mathrm{~h}^{-1} \mathrm{Mpc}^{-1}$ in the North-South direction, at the average redshift $\langle z\rangle \approx 0.054$. The X-ray emission of A 3528 itself (Schindler 1996; Gastaldello et al. 2003 ) is in the form of two sub-clumps, termed A $3528 \mathrm{~N}$ and A $3528 \mathrm{~S}$, centred on the two dominant galaxies. Despite that, the X-ray analysis showed that the two sub-clumps, and also A 3530 and A 3532, have an overall relaxed appearance in the X-ray and are classified as cool-cores (Gastaldello et al. 2003; Lakhchaura et al. 2013). Lakhchaura et al. (2013) suggested the presence of cavities around A 3532, typically found in relaxed clusters.

\section{Observations and data reduction}

\subsection{Radio data}

We observed the A 3528 and A 3558 complexes with the GMRT and with the VLA (i) to image the radio emission from the BCGs in all clusters, classify their morphology, and study their spectral properties, and (ii) to image other extended cluster radio galaxies, derive their spectral properties, and ascertain a possible connection with the cluster merger. To reach our goals, we performed observations over a wide range of frequencies and angular resolutions. Table 1 provides the details of the observations.

\subsubsection{The GMRT observations}

The A 3528 complex and the cluster A 3556 were observed on 5 and 6 April 2004, with the dual band configuration at $235 / 610 \mathrm{MHz}$. The set-up used the right (RR) polarisation at $610 \mathrm{MHz}$ and the left (LL) polarisation at $235 \mathrm{MHz}$, with bandwidths of 16 and $8 \mathrm{MHz}$, respectively. A 3558 was observed in a separate project at $325 \mathrm{MHz}$ and at $610 \mathrm{MHz}$, with $\Delta v=32 \mathrm{MHz}$. Both polarisations were recorded at the two frequencies.
The data were collected in spectral-line mode at all frequencies, that is 128 and 64 channels at 610 and $235 \mathrm{MHz}$ for project $05 \mathrm{TVa} 01$, and 256 channels at $325 \mathrm{MHz}$ and $610 \mathrm{MHz}$ for project 22_039. The calibration and data reduction were performed using the National Radio Astronomy Observatory (NRAO) Astronomical Image Processing System (AIPS) ${ }^{2}$ package. The raw data were first processed with the software flagcal (Prasad \& Chengalur 2012) to remove radio frequency interferences (RFI) and apply bandpass calibration. Then further editing, self-calibration and imaging were performed. To find a compromise between the size of the dataset and the need to minimise bandwidth smearing effects within the primary beam, after bandpass calibration the channels in each individual dataset of project $05 \mathrm{TVa} 01$ were averaged to six channels of $\approx 1 \mathrm{MHz}$ each at $235 \mathrm{MHz}$, and $\approx 2 \mathrm{MHz}$ each at $610 \mathrm{MH}$, while for project 22_039 they were averaged to 25 and 50 channels of $\approx 1$ and $0.5 \mathrm{MHz}$ each at $610 \mathrm{MHz}$ and $325 \mathrm{MHz}$ respectively. The a-priori calibration was performed using 3C286 and 1311-222 (3C283) as primary and secondary calibrators, respectively. The Baars (Baars et al. 1977) flux density scale was used. The dual band observations at $235 / 610 \mathrm{MHz}$ (project 05TVa01) were split into four pointings, centred on the three individual clusters of the A 3528 complex and on A 3556 (see Table 1), while one single pointing on A 3558 was used for project 22_039. The primary beam of each pointing is about $0.9,1.8$, and $2.5 \mathrm{deg}$ at 610,325 and $235 \mathrm{MHz}$, respectively. Therefore, at all frequencies we used a multi-facet clean to take into account the sky curvature. In addition, direction-dependent calibration was used for project 22_039 using the task PEELR in AIPS.

After a number of phase-only and one phase and amplitude self-calibration cycles, we produced the final images at each frequency for each day of observation. The three datasets on the A 3528 complex at $235 \mathrm{MHz}$ and $610 \mathrm{MHz}$ were self-calibrated, and primary-beam corrected separately and then combined to make a final mosaic. At each frequency we produced final images at full (weighting Briggs and robust 0) and at lower resolutions (weighting Briggs and robust 1 , with different $u v$ tapers depending on the frequency, and using a maximum of

\footnotetext{
2 http://aips.nrao.edu/
} 
Table 2. Observational parameters of the final images.

\begin{tabular}{lccccc}
\hline \hline Cluster & Array & $\begin{array}{c}v \\
(\mathrm{MHz})\end{array}$ & $\begin{array}{c}\text { Resolution } \\
\left({ }^{\prime \prime} \times{ }^{\prime \prime}\right)\end{array}$ & $\begin{array}{c}\text { PA } \\
\left({ }^{\circ}\right)\end{array}$ & $\begin{array}{c}\mathrm{rms} \\
\left(\mathrm{mJy} \mathrm{beam}^{-1}\right)\end{array}$ \\
\hline A35288 N & GMRT & 235 & $15.7 \times 11.8$ & 4.3 & $\sim 0.50$ \\
& & 610 & $6.2 \times 4.3$ & 24.4 & $\sim 0.10$ \\
& VLA-CnB & 8460 & $2.7 \times 1.5$ & 52.8 & $\sim 0.015$ \\
A3528 S & GMRT & 235 & $15.7 \times 11.8$ & 4.3 & $\sim 0.50$ \\
& & 610 & $6.2 \times 4.3$ & 24.4 & $\sim 0.10$ \\
& VLA-CnB & 8460 & $2.9 \times 1.4$ & 52.4 & $\sim 0.015$ \\
A3532 & GMRT & 235 & $15.7 \times 11.8$ & 4.3 & $\sim 0.50$ \\
& & 610 & $6.2 \times 4.3$ & 24.4 & $\sim 0.13$ \\
A3556 & GMRT & 235 & $16.0 \times 11.5$ & 13.7 & $\sim 0.65$ \\
& & 325 & $14.1 \times 9.5$ & 18.4 & $\sim 0.08$ \\
& & 610 & $7.0 \times 4.4$ & 31.3 & $\sim 0.09$ \\
A3558 & GMRT & 325 & $14.1 \times 9.5$ & 18.4 & $\sim 0.12$ \\
& & 610 & $9.6 \times 5.0$ & 32.8 & $\sim 0.06$ \\
\hline
\end{tabular}

Notes. The noises in the last column are an average of the whole map; "local" noises are calculated for the sigle sources and shown in the label of each figure.

$60 \%$ of the full $u v$-coverage) in search of further extensions of the radio galaxies. We did not detect further emission at low resolution at any frequency (at the sensitivity level of our images), hence the analysis and discussion in this paper is fully based on the full resolution images, which also have the best quality. The average residual amplitude errors in our data are $\leq 5 \%$ at 610 and $325 \mathrm{MHz}$, and about $8 \%$ at $235 \mathrm{MHz}$. In Table 2 we provide the relevant information on the final images for each cluster, that is, angular resolution and $1 \sigma$ noise level.

Figures 1 and 2 provide an overview of the radio emission of the A 3258 complex (at $610 \mathrm{MHz}$ ) and of the A 3558 complex (at $325 \mathrm{MHz}$ ), respectively.

The analysis and discussion in this paper is fully based on the full resolution images, which we present here, since they recover the whole extent of the radio emission in all sources and provide the highest quality.

\subsubsection{The VLA observations}

The A $3528 \mathrm{~N}$ and A3528 S complexes were observed with the VLA in the hybrid $\mathrm{CnB}$ configuration at $8.46 \mathrm{GHz}$ with two intermediate frequencies (IFs) and a total bandwidth $\Delta v=100 \mathrm{MHz}$ (see Table 1), as part of an earlier separate project. Part of those observations were published in Venturi et al. (2003). Following a standard approach, the data were edited, self-calibrated, and imaged using AIPS. The $1 \sigma$ $\mathrm{rms}$ is $\approx 0.015 \mathrm{mJy}$ beam $^{-1}$, while the residual amplitude errors are $\sim 3 \%$ (Baars flux density scale). The most relevant parameters of the final full resolution image are reported in Table 2.

\subsubsection{Complementary data}

In order to complete the spectral coverage of our analysis we added the information from the literature and archival data from the TIFR GMRT Sky Survey (TGSS) at $150 \mathrm{MHz}$, the Sydney University Molonglo Sky Survey (SUMSS) source catalogue at $843 \mathrm{MHz}$, the NRAO VLA Sky Survey (NVSS) at $1.4 \mathrm{GHz}$, and the ATCA at 1.38, 2.38, and $4.79 \mathrm{GHz}$. In Table 3 we report the resolution and the noise of the images we used and refer to the original papers.

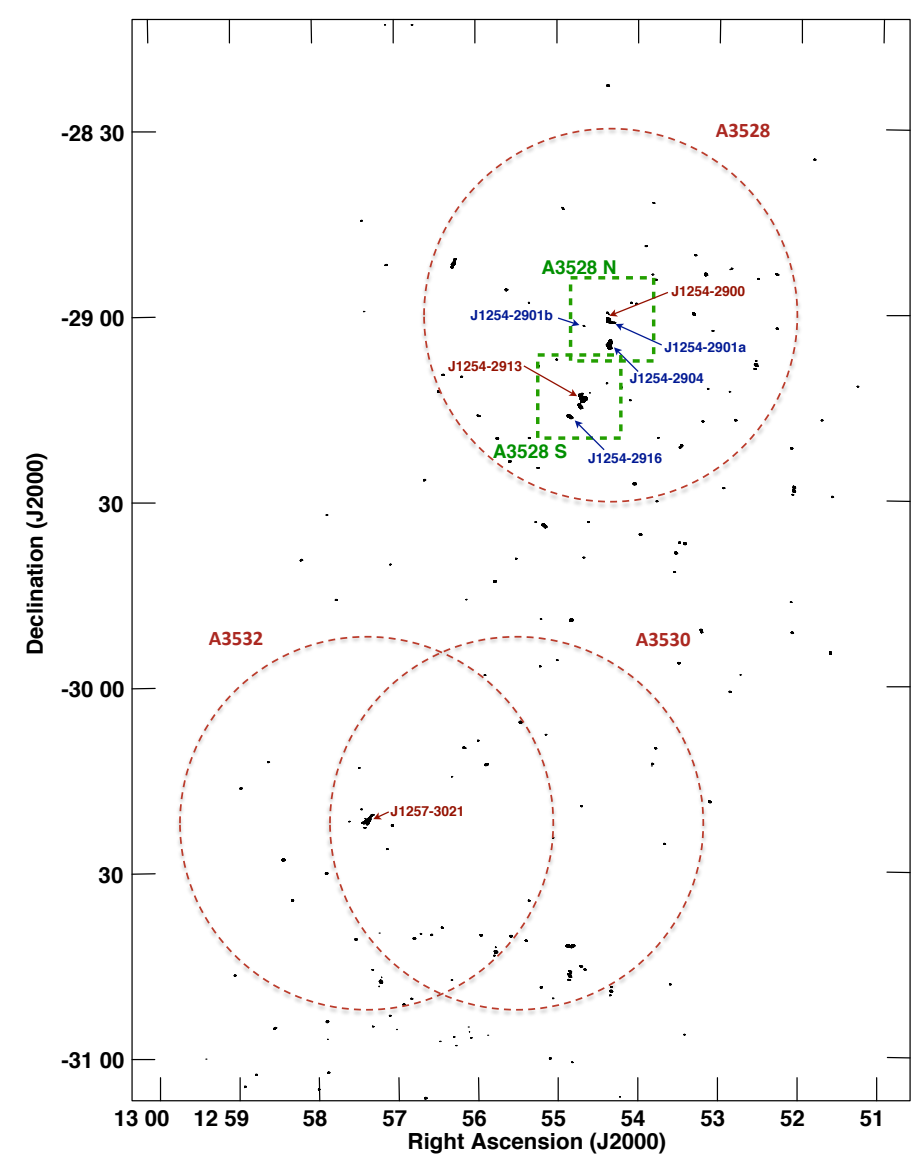

Fig. 1. Radio contours of the A 3528 complex at $610 \mathrm{MHz}$. The restoring beam is $6.8^{\prime \prime} \times 4.7^{\prime \prime}$, PA $29^{\circ}$ and the noise level of $0.16 \mathrm{mJy} \mathrm{beam}^{-1}$. The levels are $7 \sigma \times(1,2,4,8,16,32,128)$. The dashed red circles show the Abell radius of the three clusters. The BCGs are labelled in red, the tailed radio galaxies in blue. The dashed green squares represent the A $3528 \mathrm{~N}$ and A $3528 \mathrm{~S}$ sub-clusters, based on the X-ray observations (Gastaldello et al. 2003).

\subsection{New optical-NIR data and archive data}

A multi-wavelength survey of the whole Shapley supercluster (Shapley Supercluster Survey, ShaSS) was performed by Merluzzi et al. (2015) with the aim to investigate the role of mass assembly on galaxy evolution. The survey covers $23 \mathrm{deg}^{2}$ (i.e. $260 \mathrm{Mpc}^{2}$ at cluster redshift) centreed on A 3558 and combines observations in the VLT Survey Telescope (VST) ugri filters with the Visible and Infrared Survey Telescope for Astronomy (VISTA) $K$-band imaging. In the framework of this project, ShaSS has recently covered the A 3528 complex (12 $\mathrm{deg}^{2}$ in $i$ band and $3 \mathrm{deg}^{2}$ in $g$ band) providing new deep photometry for all the galaxies presented in this work. At the distance of the Shapley Concentration, the ShaSS imaging has a spatial resolution $\lesssim 1 \mathrm{kpc}$ that allows us to study the structure of the individual galaxies.

Furthermore we made use of the imaging taken with the Wide-Field Infrared Survey Explorer (WISE; Wright et al. 2010) in the three channels $\mathrm{W} 1-\mathrm{W} 3$ spanning the wavelength range 3.4-12 $\mu \mathrm{m}$.

\section{The radio galaxy population in the $\mathrm{A} 3528$ complex}

A statistical study of the radio galaxy population in the A 3528 complex was performed using ATCA and was published in Venturi et al. (2001). Here we present a detailed morphological 


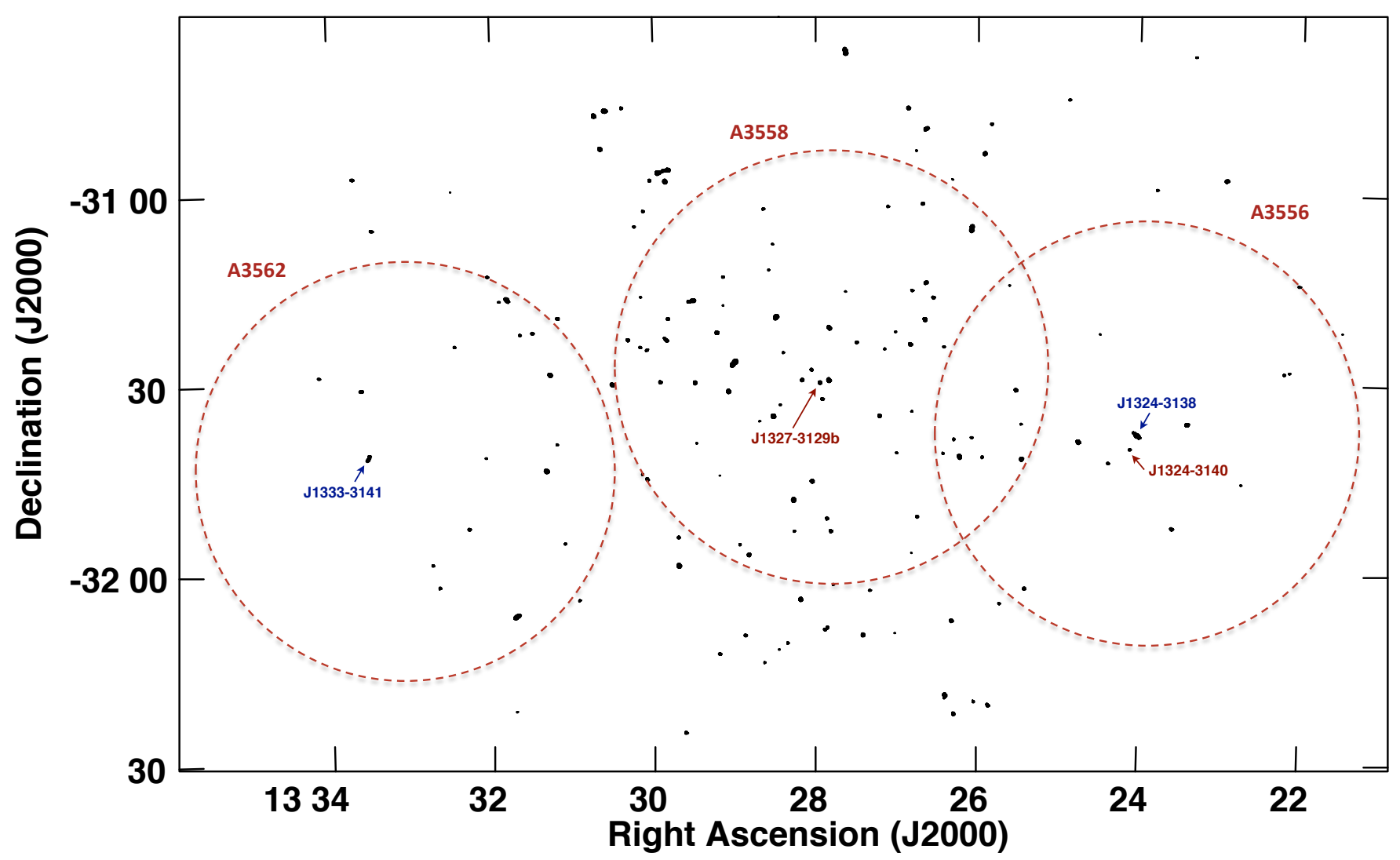

Fig. 2. Radio contours of the A 3558 complex at $325 \mathrm{MHz}$. The restoring beam is $14.05^{\prime \prime} \times 9.53^{\prime \prime}$, PA equal to $18.39^{\circ}$ and the noise level of $0.09 \mathrm{mJy}$ beam $^{-1}$. The levels are $7 \sigma \times(1,2,4,8,16,32,128)$. The dashed red circles show the Abell radius of the three clusters. The BCGs are labelled in red, the tailed radio galaxies in blue.

Table 3. Observational parameters of the literature images.

\begin{tabular}{ccccc}
\hline \hline Array & $\begin{array}{c}v \\
(\mathrm{MHz})\end{array}$ & $\begin{array}{c}\text { Resolution } \\
\left({ }^{\prime \prime} \times{ }^{\prime \prime}\right)\end{array}$ & $\begin{array}{c}\text { PA } \\
\left({ }^{\circ}\right)\end{array}$ & $\begin{array}{c}\mathrm{rms} \\
\left(\mathrm{mJy} \mathrm{beam}^{-1}\right)\end{array}$ \\
\hline TGSS-GMRT $^{a}$ & 150 & $24.0 \times 15.0$ & 30 & 9 \\
ATCA $^{b}$ & 1380 & $10.0 \times 6.0$ & 0 & 0.14 \\
ATCA $^{c}$ & 1400 & $11.8 \times 6.2$ & -0.31 & 0.56 \\
NVSS-VLA-D $^{d}$ & 1400 & $45.0 \times 45.0$ & 61 & 0.13 \\
ATCA $^{b}$ & 2380 & $6.0 \times 3.5$ & 2 & 0.15 \\
ATCA $^{c}$ & 2400 & $6.2 \times 4.2$ & 0.15 & 0.39 \\
VLA $^{g}$ & 327 & $59.0 \times 45.0$ & 80.5 & 1.9 \\
SUMSS-MOST $^{e}$ & 843 & $82.0 \times 43.0$ & 0 & 1.4 \\
ATCA $^{f}$ & 1380 & $6.0 \times 10.0$ & 0 & 0.2 \\
ATCA $^{g}$ & 1376 & $10.2 \times 6.0$ & 0.26 & 0.16 \\
ATCA $^{f}$ & 2380 & $5.3 \times 3.4$ & 0 & 0.15 \\
ATCA $^{g}$ & 4790 & $20.2 \times 10.0$ & 0 & 0.04 \\
VLA $^{g}$ & 8640 & $20.0 \times 10.0$ & 0 & 0.04 \\
\hline
\end{tabular}

Notes. The information refers to the A 3528 and A 3558 cluster complexes, on the top and on the bottom, respectively.

References. ${ }^{(a)}$ TGSS image (Intema et al. 2017); ${ }^{(b)}$ Venturi et al. (2001); ${ }^{(c)}$ Reid et al. (1998); ${ }^{(d)}$ NVSS image (http://www. cv .nrao. edu/nvss/); ${ }^{(e)}$ SUMSS catalogue (http://www.physics.usyd. edu.au/sifa/Main/SUMSS); ${ }^{(f)}$ Venturi et al. (1997); ${ }^{(g)}$ Venturi et al. (1998).

analysis of the radio emission from the BCGs and of the galaxies showing extended radio tails, while we present the spectral analysis in Sect. 6.
The sample includes the three BCGs J 1254-2900 (in A $3528 \mathrm{~N}$ ), J 1254-2913 (in A $3528 \mathrm{~S}$ ), and J 1257-3021 (A 3532) and four tailed radio galaxies: J 1254-2901a, J 12542901b, and J 1254-2904 in A $3528 \mathrm{~N}$, and J 1254-2916 in A $3528 \mathrm{~S}$. The properties of the galaxies are reported in the upper part of Table 4. The dominant galaxy of the cluster A 3530 is radio quiet, and it is reported in Table 4 for completeness.

\subsection{Radio properties of the BCGs}

\subsubsection{J 1254-2900 (A $3528 \mathrm{~N})$}

The radio emission from the $\mathrm{BCG}$ in $\mathrm{A} 3528 \mathrm{~N}$ at 1.4 and $2.3 \mathrm{GHz}$ was first reported in Reid et al. (1998) and Venturi et al. (2001), who described it as a small double radio galaxy (largest projected linear size $\sim 30 \mathrm{kpc}$ ), fully contained within the faint optical halo emission. This is confirmed also at the lower frequencies presented in this paper, where no further extension is detected either at $610 \mathrm{MHz}$ or at $235 \mathrm{MHz}$. At $8.4 \mathrm{GHz}$ the radio emission has a remarkable $\mathrm{S}$ shape. The origin of $\mathrm{S}$ - and $\mathrm{X}$-shaped radio galaxies is still debated, and one of the possibilities is the merger between two galaxies each hosting a super massive black hole (Gopal-Krishna et al. 2012). Moreover, reorientation of the radio jets has been proposed for a number of radio galaxies (Liu et al. 2018). Table 4 reports the radio power at $610 \mathrm{MHz}$, which is typical of the FRI/FRII transition value. The top left panel of Fig. 3 shows the radio emission associated with the BCG and with the head-tail source J 1254-2901a (see Sect. 4.2.1). At $235 \mathrm{MHz}$ the radio emission of the BCG blends with that of the head-tail. 
Table 4. Summary of the properties of the galaxies in our sample.

\begin{tabular}{|c|c|c|c|c|c|c|c|}
\hline $\begin{array}{l}\text { Cluster } \\
\text { name }\end{array}$ & $\begin{array}{l}\text { Galaxy } \\
\text { name }\end{array}$ & $\begin{array}{l}\mathrm{RA}_{\mathrm{J} 2000} \\
\left({ }^{\mathrm{h}} \mathrm{m} \mathrm{s}\right)\end{array}$ & $\begin{array}{c}\operatorname{Dec}_{J 2000} \\
\left({ }^{\circ}, \prime \prime \prime\right)\end{array}$ & $z$ & $\begin{array}{c}\text { Optical } \\
\text { morphology }\end{array}$ & $\begin{array}{c}\text { Radio } \\
\text { morphology }\end{array}$ & $\begin{array}{c}\log P_{610 \mathrm{MHz}} \\
\mathrm{W} \mathrm{Hz}\end{array}$ \\
\hline A $3528 \mathrm{~N}$ & J 1254-2900 & 125422.1 & -290048 & 0.0541 & $\mathrm{cD}(\mathrm{BCG})$ & $\mathrm{D}$ & 24.47 \\
\hline A $3528 \mathrm{~N}$ & J 1254-2901a & 125422.9 & -290102 & 0.0544 & Ellipt. & HT & 24.12 \\
\hline A $3528 \mathrm{~N}$ & J 1254-2904 & 125420.4 & -290409 & 0.0545 & Ellipt. & NAT & 24.41 \\
\hline A $3528 \mathrm{~N}$ & J 1254-2901b & 125440.7 & -290149 & 0.0529 & Ellipt. & NAT & 22.94 \\
\hline A $3528 \mathrm{~S}$ & J 1254-2913 & 125441.0 & -291339 & 0.0573 & $\mathrm{cD}(\mathrm{BCG})$ & WAT & 25.23 \\
\hline A $3528 \mathrm{~S}$ & J 1254-2916 & 125452.4 & -291618 & 0.0481 & Ellipt. & $\mathrm{HT}$ & 23.68 \\
\hline A 3530 & J $1255-3019$ & 125534.5 & -301950 & 0.0537 & Ellipt. & undet. & - \\
\hline A 3532 & J 1257-3021 & 125722.5 & -302145 & 0.0541 & Dumb-bell (BCG) & WAT & 25.03 \\
\hline A 3556 & J 1324-3138 & 132357.5 & -313845 & 0.0502 & Ellipt. & HT & 23.89 \\
\hline A 3556 & J $1324-3140$ & 132406.7 & -314012 & 0.0480 & $\mathrm{cD}(\mathrm{BCG})$ & $\mathrm{D}$ & 23.01 \\
\hline A 3558 & J $1327-3129 b$ & 132756.8 & -312943 & 0.0469 & $\mathrm{cD}(\mathrm{BCG})$ & unres. & 22.88 \\
\hline A 3562 & J 1333-3140 & 133334.8 & -314021 & 0.0488 & $\mathrm{cD}(\mathrm{BCG})$ & undet. & - \\
\hline A 3562 & J 1333-3141 & 133331.6 & -314101 & 0.0501 & Ellipt. & HT & $23.58^{\star}$ \\
\hline
\end{tabular}

Notes. The A 3528 complex is in the upper part, the A 3558 complex is in the lower part. D=double; HT=head tail; NAT=narrow-angle tail; WAT $=$ wide-angle tail; $\mathrm{cD}=$ central dominant. ${ }^{(\star)}$ This value was obtained from Venturi et al. (2003).

\subsubsection{J 1254-2913 (A 3528 S)}

The radio emission associated with the BCG in A3528 S is shown in the top right panel of Fig. 3, together with the head-tail J 1254-2916 (see Sect. 4.2.4). J 1254-2913 is quite complex and difficult to classify. At $8.4 \mathrm{GHz}$ the emission is resolved into three components, a "core" and two additional features (see also left panel in Fig. 8), and it is fully contained in the most central region of the host galaxy (see also Sect. 7). The emission at $610 \mathrm{MHz}$ extends on a scale slightly larger than that of the optical image, with a morphology that could be classified as wide-angle tail. At this frequency the radio emission is edge-brightened, as clear from the top right panel in Fig. 3, and its radio power is the highest in our sample (see Table 4). Some residual positive emission is visible at some distance from the BCG, suggesting the presence of more extended emission unrecoverable with the current $610 \mathrm{MHz}$ data. At $235 \mathrm{MHz}$ the radio emission encompasses the residuals detected at $610 \mathrm{MHz}$ north-east and south of the BCG, and further hints of residuals are detected in the form of filaments west of the BCG. Such residuals of very low surface brightness are clearly separated from the brightest part of the emission. They extend $\sim 100 \mathrm{kpc}$ and are suggestive of further very low surface brightness extended emission, whose origin is under investigation and will be presented in a future work.

\subsubsection{J 1257-3021 (A 3532)}

The dominant galaxy in A 3532 is a well-known dumb-bell, that is two galaxies of roughly equal brightness inside a common halo, whose radio emission has been studied at $\mathrm{GHz}$ frequencies (Gregorini et al. 1994) and at $610 \mathrm{MHz}$ (Lakhchaura et al. 2013). Only the easternmost nucleus (i.e. BCG1, see bottom panel in Fig. 3) is radio loud, with the second highest radio power among our sample (see Table 4). Radio contours from our observations are given in the bottom panel of Fig. 3. At GHz frequencies and at $610 \mathrm{MHz}$ the radio galaxy has a double morphology, with tails that bend asymmetrically to the east, suggesting a motion towards the cluster A 3530. As for J 1254-2913 in A 3528 S, the radio emission is edge brightened. At $235 \mathrm{MHz}$ the radio emission extends well beyond the optical region, in a tail elongated in the northwest direction. Such a tail shows a sharp $90^{\circ}$ bend, after which the radio emission broadens in a direction almost perpendicular to the tail itself. Unfortunately, no $8.4 \mathrm{GHz}$ VLA observations are available for this BCG, while the literature observations at $5 \mathrm{GHz}$ (Gregorini et al. 1994) do not show any morphological difference from our $610 \mathrm{MHz}$ image, and for this reason these radio contours are not shown in the bottom panel of Fig. 3.

\subsection{Tailed radio galaxies}

\subsubsection{J1254-2901a (A3528 N)}

This source is located immediately south of the BCG in A 3528 N (e.g. J1254-2900, see Sect. 4.1.1) and it is clearly a head-tail radio galaxy. The length of the tail depends on the observing frequency: it is approximately $80^{\prime \prime}$ at $235 \mathrm{MHz}$ and $610 \mathrm{MHz}(\sim 80 \mathrm{kpc}$ at source redshift), and it is considerably shorter at higher frequencies, suggesting a spectral steepening away from the nucleus. As clear from Fig. 3 (top left panel), the head of the tail remains unresolved even at $8.4 \mathrm{GHz}$, where we barely see the bifurcation of the inner part of the jets. The tail is not straight: the inner $\sim 40^{\prime \prime}$ are slightly oriented towards south, while the remaining part of the tail is aligned in the east-west direction. Overall, the tail suggests that the projected motion of the associated galaxy is pointing to the east.

\subsubsection{J1254-2901b (A $3528 \mathrm{~N})$}

The radio-optical overlay of this narrow-angle tailed radio galaxy is shown in the top left panel of Fig. 4. The radio galaxy is located $\sim 4^{\prime}$ east of the BCG of A $3528 \mathrm{~N}$, and it is very small, barely exceeding the size of the optical galaxy. It is very weak, with a radio power considerably lower than typical cluster tailed radio galaxies (Table 4). It is not visible in the VLA 8.4 GHz data presented here, most likely due to primary beam attenuation. The $235 \mathrm{MHz}$ image does not show further extension of the tail. The orientation of the tails at $610 \mathrm{MHz}$ suggests a direction of the motion towards East, similar to J 1254-2901a.

\subsubsection{J1254-2904 (A $3528 \mathrm{~N})$}

This is a very peculiar radio source, whose morphology is difficult to classify. The radio-optical overlay (top right panel of Fig. 4) clearly shows the core of the radio galaxy at $8.4 \mathrm{GHz}$, 

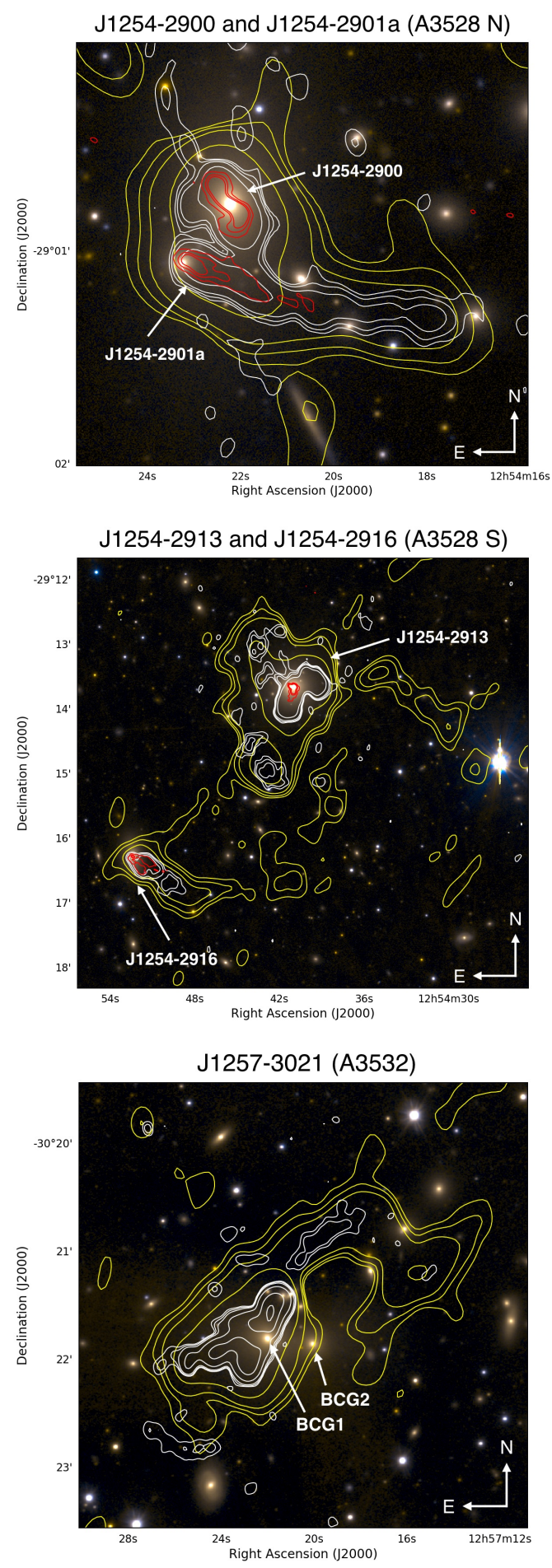

Fig. 3. VST-ACESS gri composite images with GMRT radio contours at 235 (yellow) and 610 (white) $\mathrm{MHz}$ and VLA radio contours at $8.4 \mathrm{GHz}$ (red). Top left panel: J 1254-2900 and J 1254-2901a (A 3528 N). Top right panel: J 1254-2913 and J 1254-2916 (A $3528 \mathrm{~S}$ ). The $235 \mathrm{MHz}$ contours are at $3 \sigma \times(1,2,4,8,16) \mathrm{mJy}$ beam $^{-1}$, the resolution is $16.3^{\prime \prime} \times$ $11.9^{\prime \prime}$, PA $9.5^{\circ}$, and noise level $\sigma_{235 \mathrm{MHz}}=1.26 \mathrm{mJy} \mathrm{beam}^{-1}$. The $610 \mathrm{MHz}$ contours are at $3 \sigma \times(1,2,4,8,16) \mathrm{mJy}^{-1}$ beam $^{-1}$, the resolution is $6.8^{\prime \prime} \times 4.7^{\prime \prime}$, PA $29^{\circ}$, and noise level $\sigma_{610 \mathrm{MHz}}=0.21 \mathrm{mJy} \mathrm{beam}^{-1}$. The $8.4 \mathrm{GHz}$ contours are at $3 \sigma \times(1,4,8) \mathrm{mJy} \mathrm{beam}^{-1}$, the resolution is $2.76^{\prime \prime} \times 1.59^{\prime \prime}$, PA $49.82^{\circ}$, and noise level $\sigma_{8.4 \mathrm{GHz}}=0.05 \mathrm{mJy} \mathrm{beam}^{-1}$. Bottom panel: J 1257-3021 (A 3532). The $235 \mathrm{MHz}$ contours are at $3 \sigma \times(1,2,4,8,16) \mathrm{mJy}_{\text {beam }}{ }^{-1}$, the resolution is $16.3^{\prime \prime} \times 11.9^{\prime \prime}, \mathrm{PA} 9.5^{\circ}$, and noise level $\sigma_{235 \mathrm{MHz}}=0.76 \mathrm{mJy}$ beam $^{-1}$. The $610 \mathrm{MHz}$ contours are at $3 \sigma \times(1,2,4,8,16, \ldots) \mathrm{mJy}_{\text {beam }}{ }^{-1}$, the resolution is $6.8^{\prime \prime} \times 4.7^{\prime \prime}$, PA $29^{\circ}$, and noise level $\sigma_{610 \mathrm{MHz}}=0.14 \mathrm{mJy} \mathrm{beam}^{-1}$. coincident with the optical counterpart (solid arrow in the figure), and suggesting an active nucleus. The rest of the radio emission extends southwards and could be interpreted as the lobes of a head-tail radio galaxy. At $610 \mathrm{MHz}$ (white contours) the morphology of the tail consists of three separate parts: a northern part, where the lobes are clearly visible, and well coincident with the emission at $8.4 \mathrm{GHz}$, a bottleneck and a southern extension, almost perpendicular to the direction of the lobes (see top right panel in Fig. 4). The overall extent of the radio emission is $\sim 100^{\prime \prime}$ (i.e. $\sim 90 \mathrm{kpc}$ ). This source is located in the region between the centres of A $3528 \mathrm{~N}$ and A $3528 \mathrm{~S}$, at a distance of $\sim 4^{\prime}$ and $\sim 10^{\prime}$ respectively.

An interesting possibility could be that the southern radio emission is actually part of another source. Indeed, Fig. 4 (top right panel) clearly shows that $8.4 \mathrm{GHz}$ emission is also associated with a $z=0.0704$ galaxy $\left(\mathrm{RA}_{\mathrm{J} 2000}=12^{\mathrm{h}} 54^{\mathrm{m}} 24.35^{\mathrm{s}}\right.$, $\left.\operatorname{Dec}_{\mathrm{J} 2000}=-29^{\circ} 04^{\prime} 52.6^{\prime \prime}\right)$ located just east of the southern extension (dashed arrow in the top right panel of Fig. 4). Some of the other nearby galaxies are located at a similar redshift, indicating the presence of a background group at $z \sim 0.07$ which could produce the diffuse southern emission we see at 235 and $610 \mathrm{MHz}$.

\subsubsection{J1254-2916 (A3528 S)}

This tailed radio galaxy, shown in the bottom panel of Fig. 4, is located $\sim 3^{\prime}$ south of the BCG in A 3528 S (e.g. J1254-2913, see Sect. 4.1.2 and top right panel of Fig. 3). At low frequencies the tail extends for $\sim 80^{\prime \prime}$ (i.e. $\sim 80 \mathrm{kpc}$ ), and it is longer than at high frequencies. The head of the radio galaxy points approximately north-east, providing further support to the overall "cluster weather" towards east-north-east seen both in A $3528 \mathrm{~N}$ and in A $3528 \mathrm{~S}$. The $8.4 \mathrm{GHz}$ radio contours in the overlay show that the two jets bend abruptly just out of the optical counterpart, most likely at the transition between the interstellar and intergalactic medium.

\section{The radio galaxy population in the $A 3558$ complex}

The properties of the radio galaxy population in the A 3558 complex were studied in detail at $1.4 \mathrm{GHz}$ and $2.3 \mathrm{GHz}$ with ATCA and VLA observations in Venturi et al. (1997, 2000), and Giacintucci et al. (2004). The most interesting result of those earlier works is the remarkable lack of radio galaxies in A 3558, which affects the radio luminosity function of the population of elliptical galaxies in the whole cluster complex. Here we focus our attention on the radio emission of the BCGs and of the tailed radio galaxies in the three Abell clusters, while we present their spectral analysis in Sect. 6.

The sample being studied here is formed of three BCGs, $\mathrm{J} 1324-3140$ (in A 3556), J 1327-3129b (in A 3558), and J 13333141 (in A 3562) and two tailed radio galaxies, J 1324-3138 (in A 3556) and J 1333-3141 (in A 3562). The tailed radio galaxies have already been studied in detail in Venturi et al. (1998, 2003). Here we report our earlier results (see Sects. 5.2.1 and 5.2.2) for completeness and include them in our overall discussion. The properties of the galaxies are reported in Table 4.

\subsection{Radio properties of the BCGs}

\subsubsection{J1324-3140 (A 3556)}

The radio emission associated with the BCG in A 3556 is shown in the left panel of Fig. 5, overlaid with an optical image. 

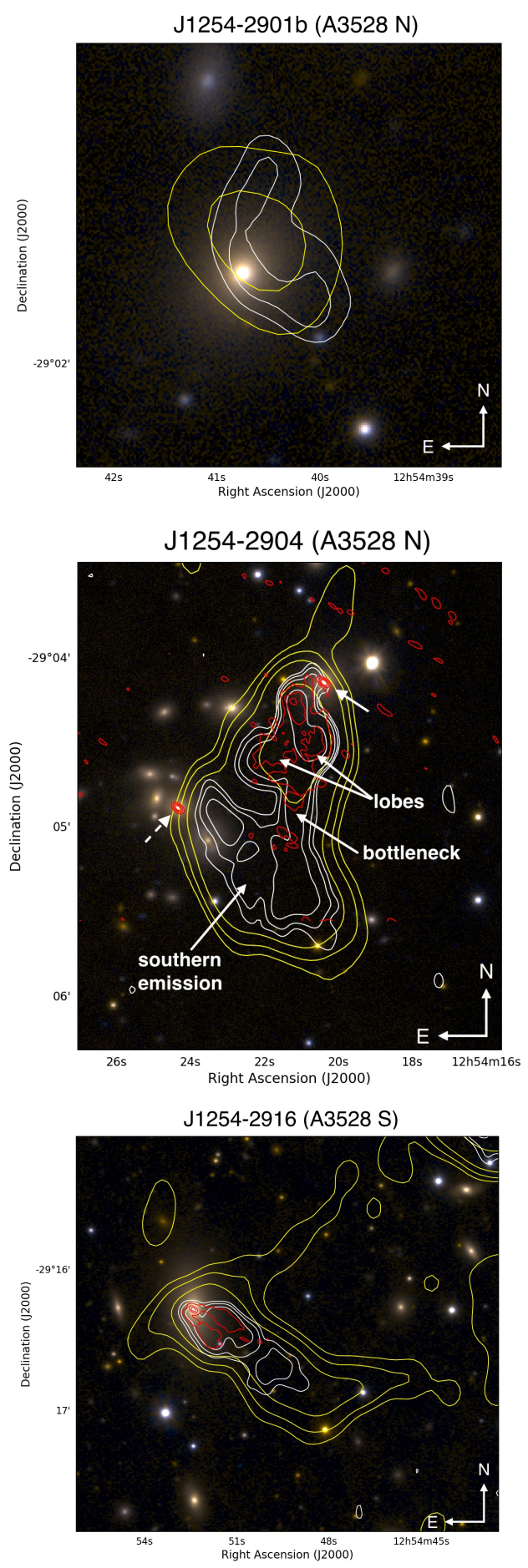

Fig. 4. VST-ACESS gri composite images with GMRT radio contours at 235 (yellow) and 610 (white) MHz and VLA radio contours at $8.4 \mathrm{GHz}$ (red). Top left panel: J 1254-2901b (A 3528 N). Top right panel: J 12542904 (A 3528 N). Bottom panel: J 1254-2916 (A 3528 S). The $235 \mathrm{MHz}$ contours are at $3 \sigma \times(1,2,4,8,16) \mathrm{mJy} \mathrm{beam}^{-1}$, the resolution is $16.3^{\prime \prime} \times$ $11.9^{\prime \prime}$, PA $9.5^{\circ}$, and noise level $\sigma_{235 \mathrm{MHz}}=1.26 \mathrm{mJy} \mathrm{beam}^{-1}$. The $610 \mathrm{MHz}$ contours are at $3 \sigma \times(1,2,4,8,16) \mathrm{mJy}^{2}$ beam $^{-1}$, the resolution is $6.8^{\prime \prime} \times 4.7^{\prime \prime}$, PA $29^{\circ}$, and noise level $\sigma_{610 \mathrm{MHz}}=0.21 \mathrm{mJy} \mathrm{beam}^{-1}$. The $8.4 \mathrm{GHz}$ contours are at $3 \sigma \times(1,4,8) \mathrm{mJy} \mathrm{beam}^{-1}$, the resolution is $2.76^{\prime \prime} \times 1.59^{\prime \prime}$, PA $49.82^{\circ}$, and noise level $\sigma_{8.4 \mathrm{GHz}}=0.05 \mathrm{mJy} \mathrm{beam}^{-1}$.

$\mathrm{J}$ 1324-3140 is a double radio galaxy, with a pair of symmetric lobes that become clearly visible only at $235 \mathrm{MHz}$ and $325 \mathrm{MHz}$. Only hints of emission from the lobes are visible at $610 \mathrm{MHz}$, suggesting a steep synchrotron spectrum. The ATCA observations at $1.4 \mathrm{GHz}$ presented in Venturi et al. (1998) show low surface brightness emission west of the nuclear component, in the region of the western lobe, which we were not able to interpret at the time. In the light of our GMRT images we can now associate this emission with the western lobe of the radio galaxy.

\subsubsection{J1327-3129b (A 3558)}

The radio emission associated with the BCG in A 3558 is shown in the right panel of Fig. 5. It is a compact and unresolved source at all frequencies of our observations. The radio emission is fully confined into the faint optical galaxy halo. It is the weakest radio galaxy in our sample, as is clear from Table 4.

\subsubsection{J1333-3140 (A3562)}

The cluster A 3562 was studied in detail in Venturi et al. (2003) and Giacintucci et al. (2005), by means of GMRT and ATCA observations from $235 \mathrm{MHz}$ to $8.4 \mathrm{GHz}$. The cluster hosts a radio halo, whose origin has been interpreted in the light of the ongoing merging events in the region between A 3558 and A 3562. Our $325 \mathrm{MHz}$ image (Fig. 2) is consistent with our earlier studies (Venturi et al. 2000) and confirms that the BCG in A 3562 is radio quiet (see also the optical-radio overlay in the bottom panel in Fig. 5; the white arrow indicates the BCG in A 3562).

\subsection{Tailed radio galaxies}

\subsubsection{J1324-3138 (A3556)}

J 1324-3138 is shown in the left panel of Fig. 5. It lies very close to the centre of A 3556, at a projected distance of only $\sim 2.5^{\prime}$ from the BCG (e.g. J 1324-3140). It was studied in detail in Venturi et al. (1998), where they concluded that the source is most likely a head-tail radio galaxy whose nuclear emission has switched off, based on the spectral analysis. None of our observations is able to resolve the nuclear component and the inner jets (if present). Our new GMRT observations show a similar extent and morphology of those earlier studies, ruling out further extended emission at low frequency.

\subsubsection{J1333-3141 (A3562)}

This head-tail radio galaxy was studied in detail from $235 \mathrm{MHz}$ to $8.4 \mathrm{GHz}$ with GMRT and ATCA observations (Venturi et al. 2003; Giacintucci et al. 2005). It is located at a projected distance of $\sim 1^{\prime}$ south of the centre of A 3562, the cluster that lies at the western end of the A 3558 cluster complex (see Fig. 2). The length of the tail is $\sim 1^{\prime}$ (i.e. $\sim 60 \mathrm{kpc}$ at cluster's redshift), before it merges in the radio halo. It is not straight, but it smoothly bends from east to west, suggesting a possible orbital motion around the cluster BCG (see Fig. 6 in Venturi et al. 2003 and bottom panel in Fig. 5). No further imaging or analysis is presented in this paper, but we review the literature information here to help the discussion that will be presented in Sect. 8.

\section{Spectral analysis}

In order to study the nature of the radio emission of the galaxies in our sample, and possibly identify different evolutionary stages in their life cycle, we performed a spectral study of each radio galaxy in the frequency range $150 \mathrm{MHz}-8.46 \mathrm{GHz}$, by means of the information at six to nine different frequencies (see 

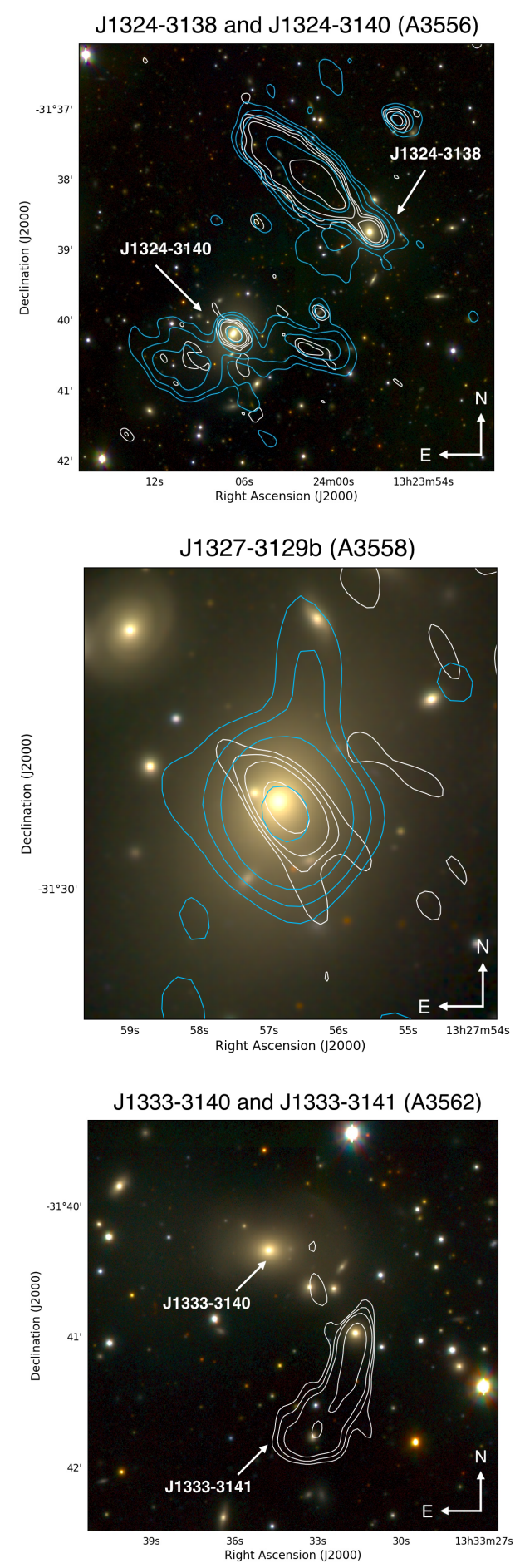

Fig. 5. VST-ACESS gri composite images with GMRT radio contours at 325 (blue) and 610 (white) MHz. Top left panel: J 13243138 and $\mathrm{J} 1324-3140$ (A 3556). The $325 \mathrm{MHz}$ contours are at $3 \sigma \times$ $(1,2,4,8,16) \mathrm{mJy}$ beam ${ }^{-1}$, the resolution is $16.3^{\prime \prime} \times 11.89^{\prime \prime}, \mathrm{PA} 12.29^{\circ}$, and noise level $\sigma_{325 \mathrm{MHz}}=0.20 \mathrm{mJy}^{-1}$ beam $^{-1}$. The $610 \mathrm{MHz}$ contours are at $3 \sigma \times(2,4,8,16) \mathrm{mJy}^{-1}$ beam $^{-1}$, the resolution is $12.86^{\prime \prime} \times 7.2^{\prime \prime}$, PA $41.78^{\circ}$, and noise level $\sigma_{610 \mathrm{MHz}}=0.08 \mathrm{mJy}$ beam $^{-1}$. Top right panel: J 1327-3129b (A 3558). The $325 \mathrm{MHz}$ contours are at $3 \sigma \times$ $(2,4,8,16) \mathrm{mJy}$ beam $^{-1}$, the resolution is $16.3^{\prime \prime} \times 11.89^{\prime \prime}$, PA $12.29^{\circ}$, and noise level $\sigma_{325 \mathrm{MHz}}=0.25 \mathrm{mJy}^{-1}$ beam $^{-1}$. The $610 \mathrm{MHz}$ contours are at $3 \sigma \times(2,4,8,16) \mathrm{mJy}_{\text {beam }}{ }^{-1}$, the resolution is $10.98^{\prime \prime} \times 5.59^{\prime \prime}$, PA $34.16^{\circ}$, and noise level $\sigma_{610 \mathrm{MHz}}=0.14 \mathrm{mJy}_{\text {beam }}{ }^{-1}$. Bottom panel: $\mathrm{J} 1333-3140$ and $\mathrm{J} 1333-3141$ (A 3562). The $610 \mathrm{MHz}$ contours are at $3 \sigma \times(2,4,8,16) \mathrm{mJy}$ beam ${ }^{-1}$, the resolution is $8^{\prime \prime} \times 6^{\prime \prime}, \mathrm{PA} 0^{\circ}$, and noise level $\sigma_{610 \mathrm{MHz}}=0.08 \mathrm{mJy}_{\text {beam }}^{-1}$.
Tables 5 and 6). Our procedure and results are reported in the next subsections. The spectra and best-fit results from radiation loss models for the BCGs and for the tailed radio galaxies are reported in Figs. 6 and 7.

\subsection{Integrated spectra}

The total integrated spectrum for all galaxies in the sample was derived using the flux density measurements reported in Tables 5 and 6. The tables report three sets of measurements. In particular:

- The total flux density measurements at each frequency were obtained by means of the AIPS task TVSTAT integrating within the $3 \sigma$ contour level. We took the $235 \mathrm{MHz}$ emitting volume as the reference, and we measured the flux density at each frequency over the same volume, to account for the different sensitivities of our images. The only exception is $\mathrm{J}$ 1324-3140 (BCG in A 3556) for which we used the best available image at $325 \mathrm{MHz}$.

- We refer to the flux density of the "central" region, meaning the emission that is clearly associated with the optical counterpart. We are aware that this definition is not very rigorous. In a couple of cases, as in J 1324-3138 and J 13243140 (radio tail and BCG in A 3556, respectively), it is coincident with the radio core, while in other cases it refers to the full radio emission except for the extended component, which is clearly separated, as in the case of J 1254-2901a and J 1254-2904 (radio tails in A 3528 N), J 1254-2913 (BCG in A $3528 \mathrm{~S}$ ) and $\mathrm{J} 1257-3021$ (BCG in A 3532). The strong edge brightening of the latter two sources provides a natural definition of this part of the radio emission. We point out that due to the average resolution of our images, in most cases the compact core is clearly imaged only at $8.4 \mathrm{GHz}$, as is the case of J 1254-2904 (radio tail in A 3528 N), J 1254-2913, and $\mathrm{J}$ 1254-2916 (BCG and radio tail in A $3528 \mathrm{~S}$, respectively), while it blends with the rest of the "central" region at any other frequency. For this reason a spectral study of the core is not feasible.

- Finally, the flux density of the diffuse emission is obtained by subtraction of the central flux density from the total one. The only exceptions are J 1257-3021 (BCG in A 3532) and $\mathrm{J}$ 1324-3140 (BCG in A 3556), where the values for the central and the diffuse component were obtained individually, as they can be easily separated in our images.

For better clarity, the insets in Figs. 6 and 7 show the region corresponding to total emission (black contours) and to the central region (single red contour). The flux density error is derived as the sum of the contribution of the thermal noise of the image and of the residual calibration error. The latter is dominating in our cases (Chandra et al. 2004).

\subsubsection{Spectral properties of the BCGs}

Here below we provide further details for each source.

$J 1254-2900$ (A $3528 N$ ). No diffuse emission is detected for this radio galaxy (see Sect. 4.1.1). The spectrum is well fitted by a single power law with $\alpha_{235 \mathrm{MHz}}^{8.4 \mathrm{GHz}}=0.83_{-0.11}^{+0.03}$, typical of an active source emitting via synchrotron radiation.

$J 1254-2913$ (A 3528 S). The spectrum of the central region is well fitted by a single power law, with $\alpha_{235 \mathrm{MHz}}^{8.4 \mathrm{GHz}}=1.01_{-0.11}^{+0.03}$. The spectral index of the total emission up to $610 \mathrm{MHz}$ was obtained both with and without the $150 \mathrm{MHz}$ flux density measurement. In both cases we obtained $\alpha \sim 1.2$. Since the total emission is dominated by the central region, the similarity of the two spectra 
Table 5. Flux measurements for the sample of the BCGs. The information on the observations (e.g. beam and literature references) are provided in Tables 2 and 3.

\begin{tabular}{|c|c|c|c|c|c|c|c|}
\hline $\begin{array}{l}\text { Radio } \\
\text { galaxy }\end{array}$ & $\begin{array}{c}\text { Cluster } \\
\text { name }\end{array}$ & Telescope & $\begin{array}{c}v \\
(\mathrm{MHz})\end{array}$ & $\begin{array}{c}S_{\text {tot }} \\
(\mathrm{mJy})\end{array}$ & $\begin{array}{c}S_{\text {central }} \\
(\mathrm{mJy})\end{array}$ & $\begin{array}{c}S_{\text {diff }} \\
(\mathrm{mJy})\end{array}$ & Ref. \\
\hline \multirow{5}{*}{ J 1254-2900 } & \multirow{5}{*}{ A $3528 \mathrm{~N}$} & GMRT & 235 & $996.1 \pm 79.7$ & & & This work \\
\hline & & GMRT & 610 & $428.1 \pm 21.4$ & & & This work \\
\hline & & ATCA & 1380 & $230.9 \pm 6.9$ & & & (Venturi et al. 2001) \\
\hline & & ATCA & 2380 & $142.4 \pm 4.3$ & & & (Venturi et al. 2001) \\
\hline & & VLA & 8400 & $50.5 \pm 1.5$ & & & This work \\
\hline \multirow{8}{*}{ J 1254-2913 } & \multirow{8}{*}{ A $3528 \mathrm{~S}$} & GMRT & 150 & $14491.0 \pm 2898.2$ & & & TGSS image \\
\hline & & GMRT & 235 & $7291.4 \pm 583.3$ & $4714.5 \pm 377.2$ & $2576.9 \pm 694.6$ & This work \\
\hline & & GMRT & 610 & $2159.1 \pm 108.0$ & $1956.7 \pm 97.8$ & $202.4 \pm 145.8$ & This work \\
\hline & & MOST & 843 & $1970.0 \pm 59.1$ & & & SUMSS image \\
\hline & & ATCA & 1380 & & $936.7 \pm 28.1$ & & (Venturi et al. 2001) \\
\hline & & VLA & 1400 & $1069.1 \pm 32.1$ & & $132.4 \pm 42.6^{a}$ & NVSS image \\
\hline & & ATCA & 2380 & & $538.4 \pm 16.2$ & & (Venturi et al. 2001) \\
\hline & & VLA & 8400 & & $125.3 \pm 3.8$ & & This work \\
\hline \multirow{7}{*}{ J $1257-3021$} & \multirow{7}{*}{ A 3532} & GMRT & 150 & $8655.9 \pm 1410.1$ & $6802.4 \pm 1360.5$ & $1853.5 \pm 370.7$ & TGSS image \\
\hline & & GMRT & 235 & $4591.9 \pm 336.0$ & $4251.3 \pm 334.9$ & $340.5 \pm 27.2$ & This work \\
\hline & & GMRT & 610 & $1522.0 \pm 74.2$ & $1484.0 \pm 74.2$ & $38.0 \pm 1.9$ & This work \\
\hline & & MOST & 840 & $1773.0 \pm 53.2$ & & & SUMSS image \\
\hline & & ATCA & 1380 & & $1056.5 \pm 31.7$ & & (Venturi et al. 2001) \\
\hline & & VLA & 1400 & $1061.0 \pm 31.8$ & $4.5 \pm 44.4^{a, b}$ & & NVSS image \\
\hline & & ATCA & 2380 & & $651.7 \pm 19.6$ & & (Venturi et al. 2001) \\
\hline \multirow{7}{*}{ J $1324-3140$} & \multirow{7}{*}{ A 3556} & GMRT & 235 & $77.1 \pm 6.2$ & $18.6 \pm 1.5$ & $58.5 \pm 6.4$ & This work \\
\hline & & GMRT & 325 & $84.6 \pm 4.2$ & $16.8 \pm 0.8$ & $67.8 \pm 4.3$ & This work \\
\hline & & GMRT & 610 & $18.7 \pm 0.9$ & $10.9 \pm 0.5$ & $7.8 \pm 1.0$ & This work \\
\hline & & MOST & 843 & $16.7 \pm 0.5$ & & & SUMSS image \\
\hline & & ATCA & 1380 & & $7.8 \pm 0.2$ & & (Venturi et al. 1997) \\
\hline & & ATCA & 2380 & & $7.4 \pm 0.2$ & & (Venturi et al. 1997) \\
\hline & & ATCA & 4790 & & $3.0 \pm 0.1$ & & (Venturi et al. 2008) \\
\hline \multirow{5}{*}{ J 1327-3129b } & \multirow{5}{*}{ A 3558} & GMRT & 235 & $19.1 \pm 1.5$ & & & This work \\
\hline & & GMRT & 325 & $17.9 \pm 1.4$ & & & This work \\
\hline & & GMRT & 610 & $14.6 \pm 0.7$ & & & This work \\
\hline & & ATCA & 1380 & $6.2 \pm 0.2$ & & & (Venturi et al. 1997) \\
\hline & & ATCA & 2380 & $1.5 \pm 0.1$ & & & (Venturi et al. 1997) \\
\hline
\end{tabular}

Notes. The flux density of the diffuse emission is the difference between the total flux density and the central emission, i.e. the emission contained within the optical counterpart, which is encompassed in the region highlighted in red in Figs. 6 and 7. The resolution of TGSS does not allow to separate J 1254-2900 and J 1254-2901a (A 3528 N). J 1254-2900 (A 3528 N) and J 1327-3129b (A 3558) do not show extended emission, and we provide only the total flux density. For J 1257-3021 (A 3532) we measured the flux density of the diffuse and central emission separately, and the total one as a sum of the two. ${ }^{(a)}$ The diffuse emission was calculated by difference between the VLA (total region) and the ATCA (central region) observation. ${ }^{(b)}$ Upper limit.

is not surprising. The spectral index of the diffuse emission is steeper, with $\alpha_{235 \mathrm{MHz}}^{1.4 \mathrm{GHz}}=1.77_{-0.56}^{+0.65}$. We note that our flux density measurement at $610 \mathrm{MHz}$ is well below this power law. Even considering this value a lower limit (in the light of the hints of extended emission at the sensitivity level of our observations), the difference between the spectrum of the central emission, the steep value of the diffuse emission, and the sharp morphological transition and surface brightness distribution between the central and diffuse part of the radio emission cannot be ignored.

$J$ 1257-3021 (A 3532). The spectrum of the central region of the radio galaxy is consistent with an active structure $\left(\alpha_{235 \mathrm{MHz}}^{2.38 \mathrm{GHz}}=0.71_{-0.06}^{+0.02}\right)$. The spectral index of the diffuse emission in the north-west direction, observed at 150,235 and $610 \mathrm{MHz}$ is $\alpha_{150 \mathrm{MHz}}^{610 \mathrm{MHz}}=2.63_{-0.37}^{+0.43}$. As in the case of J 1254-2913 (in A $3528 \mathrm{~S}$ ) the sharp morphological transition and surface brightness drop between the central region and the diffuse emission is noticeable.
$J 1324-3140$ (A 3556). Beyond the compact component associated with the nucleus of the BCG, the low frequency observations show the presence of a pair of symmetric lobes, which are well imaged only at $325 \mathrm{MHz}$, most likely due to the fact that the $u-v$ coverage of our snapshot observations at $610 \mathrm{MHz}$ and $235 \mathrm{MHz}$ is inadequate to image such low brightness features. This is suggested by the scatter in the flux density of the lobes between $235 \mathrm{MHz}$ and $610 \mathrm{MHz}$, which does not allow any estimate of the spectral index, and the flux density values at these two frequencies should be considered lower limits. The spectral behaviour of the central region is very different. Despite some scattering, its spectrum is very flat, $\alpha_{235 \mathrm{MHz}}^{5 \mathrm{GHz}}=0.57_{-0.11}^{+0.04}$. It is possible that the nuclear region of J 1324-3140 is variable: our measurements span a time interval of almost 30 years, and variability in the source would indeed reproduce the observed scatter. We point out that calibration problems at any of the frequencies are ruled out by the consistency of the flux density measurements of 
G. Di Gennaro et al.: Cosmic dance in the Shapley Concentration Core. I.

Table 6. Flux measurements for the sample of the tailed galaxies.

\begin{tabular}{|c|c|c|c|c|c|c|c|}
\hline $\begin{array}{l}\text { Radio } \\
\text { galaxy }\end{array}$ & $\begin{array}{c}\text { Cluster } \\
\text { name }\end{array}$ & Telescope & $\begin{array}{c}v \\
(\mathrm{MHz})\end{array}$ & $\begin{array}{c}S_{\text {tot }} \\
(\mathrm{mJy})\end{array}$ & $\begin{array}{c}S_{\mathrm{c}} \\
(\mathrm{mJy})\end{array}$ & $\begin{array}{c}S_{\text {diff }} \\
(\mathrm{mJy})\end{array}$ & Ref. \\
\hline \multirow{5}{*}{ J 1254-2901a } & \multirow{5}{*}{ A $3528 \mathrm{~N}$} & GMRT & 235 & $349.4 \pm 28.0$ & $239.9 \pm 19.2$ & $109.5 \pm 34.0$ & This work \\
\hline & & GMRT & 610 & $186.6 \pm 9.3$ & $140.0 \pm 7.0$ & $46.6 \pm 11.6$ & This work \\
\hline & & ATCA & 1380 & & $110.5 \pm 3.3$ & & (Venturi et al. 2001) \\
\hline & & ATCA & 2380 & & $57.9 \pm 1.7$ & & (Venturi et al. 2001) \\
\hline & & VLA & 8400 & & $22.1 \pm 0.7$ & & This work \\
\hline \multirow{2}{*}{ J 1254-2901b } & \multirow{2}{*}{ A $3528 \mathrm{~N}$} & GMRT & 235 & $22.7 \pm 1.8$ & & & This work \\
\hline & & GMRT & 610 & $13.1 \pm 0.7$ & & & This work \\
\hline \multirow{8}{*}{ J 1254-2904 } & \multirow{8}{*}{ A $3528 \mathrm{~N}$} & GMRT & 150 & $1463.7 \pm 292.7$ & $607.4 \pm 121.5$ & $856.3 \pm 316.9$ & TGSS image \\
\hline & & GMRT & 235 & $926.6 \pm 74.1$ & $416.1 \pm 33.3$ & $510.6 \pm 81.2$ & This work \\
\hline & & GMRT & 610 & $342.8 \pm 17.1$ & $221.4 \pm 11.1$ & $121.4 \pm 20.4$ & This work \\
\hline & & MOST & 843 & $519.0 \pm 15.6$ & & & SUMSS image \\
\hline & & ATCA & 1380 & $295.9 \pm 8.9$ & & & (Venturi et al. 2001) \\
\hline & & VLA & 1400 & $311.7 \pm 9.4$ & & & NVSS image \\
\hline & & ATCA & 2380 & $142.8 \pm 4.3$ & $126.2 \pm 3.8$ & $16.6 \pm 5.7$ & (Venturi et al. 2001) \\
\hline & & VLA & 8400 & & $30.0 \pm 1.0$ & & This work \\
\hline \multirow{7}{*}{ J 1254-2916 } & \multirow{7}{*}{ A $3528 \mathrm{~S}$} & GMRT & 235 & $259.3 \pm 20.7$ & $132.8 \pm 10.6$ & $126.5 \pm 23.3$ & This work \\
\hline & & GMRT & 610 & $149.1 \pm 7.5$ & $76.5 \pm 3.8$ & $72.6 \pm 8.4$ & This work \\
\hline & & MOST & 843 & $124.0 \pm 3.7$ & & & SUMSS image \\
\hline & & ATCA & 1380 & \multirow{4}{*}{$85.8 \pm 2.6$} & $62.1 \pm 1.9$ & & (Venturi et al. 2001) \\
\hline & & VLA & 1400 & & & $23.7 \pm 3.2^{a}$ & NVSS image \\
\hline & & ATCA & 2380 & & $49.6 \pm 1.5$ & & (Venturi et al. 2001) \\
\hline & & VLA & 8400 & & $15.3 \pm 0.5$ & & This work \\
\hline \multirow{11}{*}{ J 1324-3138 } & \multirow{11}{*}{ A 3556} & GMRT & 235 & $278.3 \pm 22.3$ & $9.1 \pm 0.7$ & $269.2 \pm 22.3$ & This work \\
\hline & & VLA & 327 & $230 \pm 11.5$ & & & (Venturi et al. 1998) \\
\hline & & GMRT & 325 & $254 \pm 12.7$ & $8.1 \pm 0.4$ & $245.9 \pm 12.7$ & This work \\
\hline & & GMRT & 610 & $122.7 \pm 6.1$ & $6.2 \pm 0.2$ & $119.5 \pm 6.1$ & This work \\
\hline & & MOST & 843 & $80.2 \pm 2.4$ & & & (Venturi et al. 1998) \\
\hline & & ATCA & 1380 & $41 \pm 1.2$ & $2.1 \pm 0.1$ & $38.9 \pm 1.2$ & (Venturi et al. 1997) \\
\hline & & ATCA & 1400 & $43.1 \pm 1.3$ & $2.7 \pm 0.1$ & $40.4 \pm 1.3$ & ATCA archive image \\
\hline & & ATCA & 2380 & $21.0 \pm 0.6$ & $1.7 \pm 0.1$ & $19.3 \pm 0.6$ & (Venturi et al. 1997) \\
\hline & & ATCA & 4790 & $7.3 \pm 0.3$ & $0.5 \pm 0.03$ & $6.8 \pm 0.3$ & (Venturi et al. 1998) \\
\hline & & ATCA & 5000 & $6.8 \pm 0.2$ & $0.4 \pm 0.01$ & $6.5 \pm 0.2$ & ATCA archive image \\
\hline & & ATCA & 8640 & $2.2 \pm 0.1$ & $0.1 \pm 0.01$ & $2.1 \pm 0.1$ & (Venturi et al. 1998) \\
\hline
\end{tabular}

Notes. The information on the observations (e.g. beam and literature references) are provided in Tables 2 and 3. The diffuse emission is the difference between the total flux density and the emission from the central components. ${ }^{(a)}$ The diffuse emission was calculated by difference between the VLA (total region) and the ATCA (central region) observation.

the other sources in the field, and in particular $\mathrm{J} 1324-3138$ (in A 3556, see Sect. 6.1.2).

$J 1327-3129 b$ (A 3558). This source is all confined within the nucleus of the BCG, and its spectrum shows a peculiar trend. It is almost flat below $610 \mathrm{MHz}$, then it steepens dramatically above this frequency. The spectral index has been calculated into three frequency intervals, $\alpha_{235 \mathrm{MHz}}^{610 \mathrm{MHz}}=0.28 \pm 0.07$ $\alpha_{610 \mathrm{MHz}}^{1.4 \mathrm{GHz}}=1.03 \pm 0.09$ and $\alpha_{1.4 \mathrm{GHz}}^{2.4 \mathrm{GHz}}=2.63 \pm 0.50$. We also re-analysed archival VLA data at $4.85 \mathrm{GHz}(\mathrm{CnB}$ configuration, beam size of $\sim 11^{\prime \prime}$ resolution). At this frequency we measured a radio flux density of $1.2 \pm 0.2 \mathrm{mJy}$, which led to a spectral index of $\alpha_{1.4 \mathrm{GHz}}^{4.9 \mathrm{GHz}}=1.31 \pm 0.14$. Considering that the source is undetected in the TGSS, the overall spectrum is suggestive of a concave shape, typical of compact steep spectrum sources (CSS; see Fanti et al. 1995) or megahertz peaked sources (MPS; Coppejans et al. 2017), that is active galactic nuclei in the early stages of their radio activity. The steep spectrum in the optically thin region suggests the presence of a population of old electrons, although a steeper spectrum in the optically thin region has been recently found for the "young" CSS J1613+4223 (Dallacasa \& Orienti 2016, $\alpha \sim 1.6$ ).

\subsubsection{Spectral properties of the tailed radio galaxies}

Following the same procedure as for the BCGs, we performed the spectral analysis for the tailed radio sources, by means of the integrated fluxes in Table 6.

J 1254-2901a (A 3528 N). The central region, which includes the core and inner part of the tail, has a spectrum with $\alpha_{235 \mathrm{MHz}}^{8.4 \mathrm{MHz}}=$ $0.67_{-0.10}^{+0.03}$. At low frequencies the tail extends to $\sim 80 \mathrm{kpc}$ (see Sect. 4.2.1) and its spectrum steepens from $\alpha_{235 \mathrm{MHz}}^{610 \mathrm{MHz}}=0.56 \pm 0.10$ to $\alpha_{235 \mathrm{MHz}}^{610 \mathrm{MHz}}=0.89 \pm 0.42$, consistent with what is usually seen in tailed radio galaxies (e.g. Pizzo \& de Bruyn 2009; Stroe et al. 2013).

$J 1254-2901 b(A 3528 N)$. The spectrum of this tail, in the very limited range available here, is $\alpha_{235 \mathrm{MHz}}^{610 \mathrm{MH}}=0.58 \pm 0.10$, again typical of an active source.

$J 1254-2904$ (A $3528 N$ ). The active nucleus associated with the optical counterpart is clearly separated from the rest of the emission only at $8.4 \mathrm{GHz}$, hence no estimate of its spectral index can be derived from our images. The central region is detected all the way up to $8.4 \mathrm{GHz}$, with a spectral index $\alpha_{150 \mathrm{MHz}}^{8.4 \mathrm{GHz}}=$ $0.72_{-0.08}^{+0.03}$. Conversely the spectrum of the southern extension is 

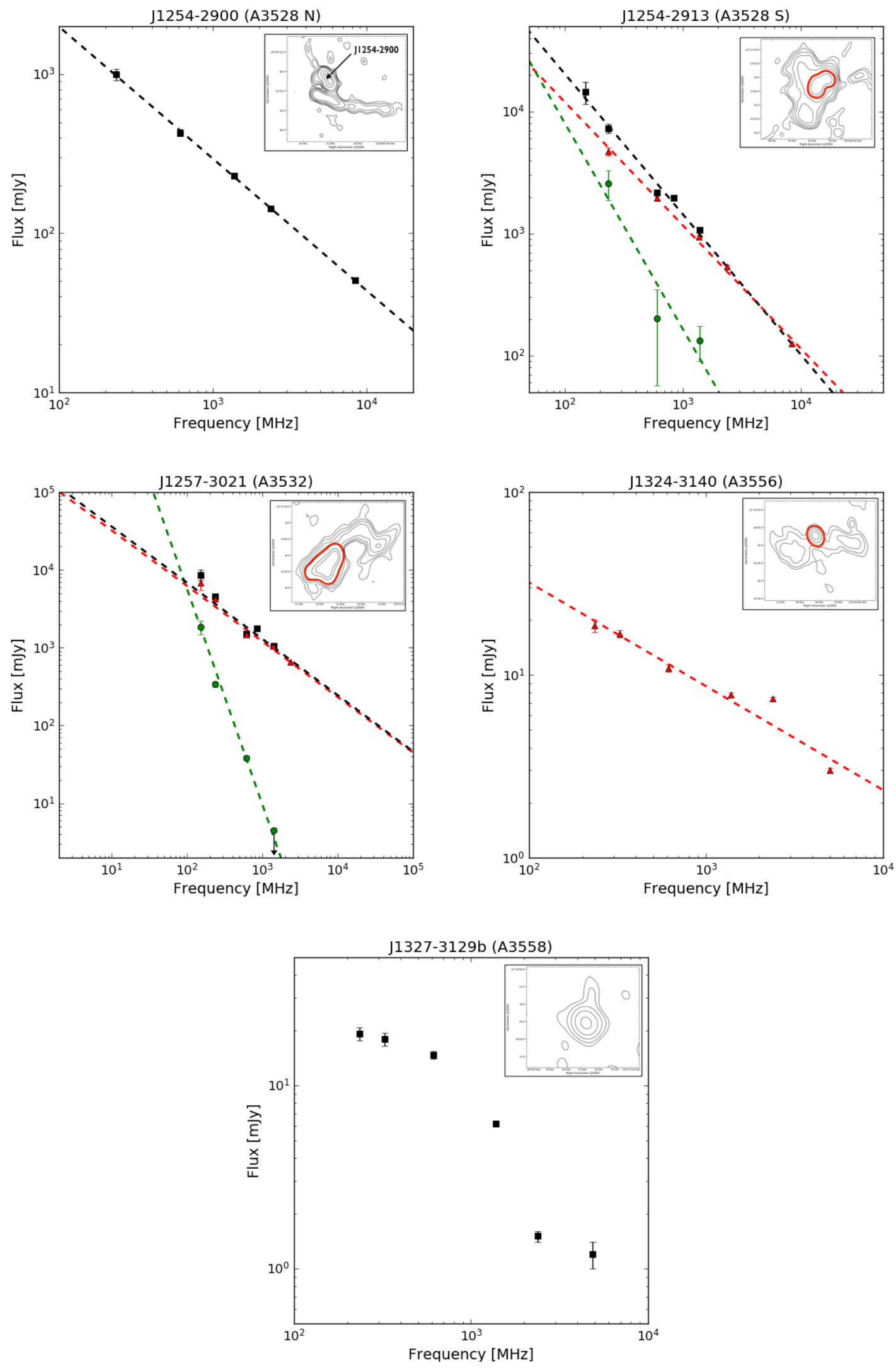

Fig. 6. Integrated radio spectra for the total (black) central (red), and diffuse (green) emission for the BCGs sample. The contours of the radio galaxy are displayed in the top right corner of each panel. The red line shows the region that has been taken as "central" emission. The value at $1.4 \mathrm{GHz}$ in the J 1257-3021 (A 3532) spectrum is an upper limit.

considerably steeper, $\alpha_{150 \mathrm{MHz}}^{2.38 \mathrm{GHz}}=1.45_{-0.21}^{+0.05}$. The reason for the abrupt change in the morphological and spectral properties of this radio galaxy south of the bottleneck is unclear. It is becoming evident that the interplay between the radio plasma in 

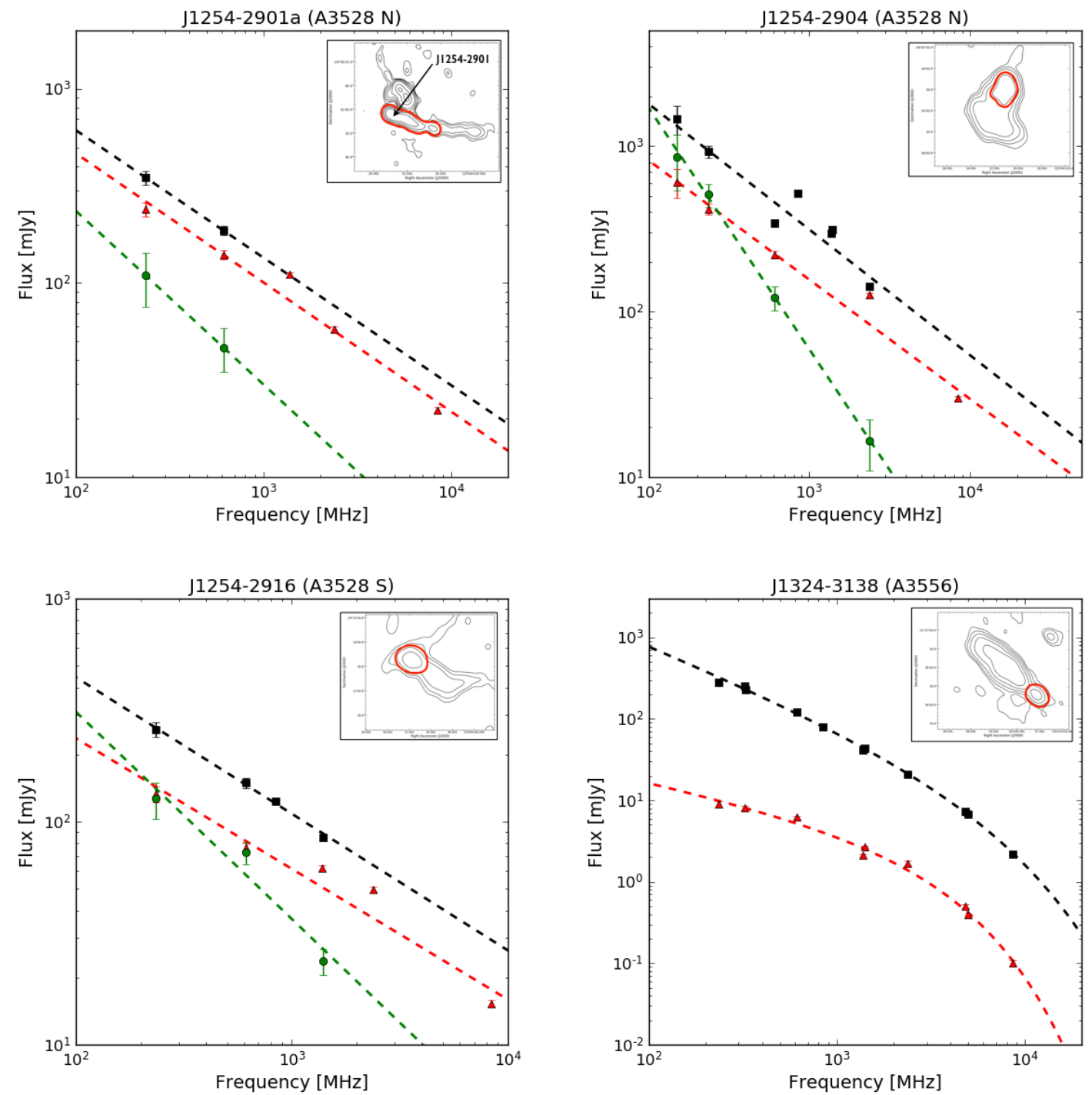

Fig. 7. Integrated radio spectra for the total (black) central (red), and diffuse (green) emission for the tails sample. The contours of the radio galaxy are displayed in the top right corner of each spectrum. The red line shows the region that has been taken as "central" emission.

galaxy clusters and the presence of perturbations in the intracluster medium (such as for instance shocks) may be common (i.e. Shimwell et al. 2016; de Gasperin et al. 2017). Considering that J 1254-2904 is located in the region between A $3528 \mathrm{~N}$ and A $3528 \mathrm{~S}$, the possibility that the southern extension is old revived radio plasma is intriguing. An alternative possibility is that this region is actually aged radio plasma associated with the $z=0.0704$ galaxy located just outside the radio contours (see Sect. 4.2.3 and Fig. 4).

$J$ 1254-2916 (A 3528 S). As for J 1254-2901a (in A $3528 \mathrm{~N}$ ), the length of the tail increases with decreasing frequency (see Fig. 4). At low frequencies ( 235 and $610 \mathrm{MHz}$ ) the spectral index is $\alpha_{235 \mathrm{MHz}}^{610 \mathrm{MHz}}=0.98_{-0.22}^{+0.21}$. The central region is well described by a power law, $\alpha_{235 \mathrm{MHz}}^{8.4 \mathrm{GHz}}=0.59_{-0.06}^{+0.03}$.

For the analysis of $\mathrm{J} 1324-3138$ (in A 3556) and $\mathrm{J} 1333-3141$ (in A 3562) we refer to works from the literature (Venturi et al. 1998, 2003, respectively). For J 1324-3138 (in A 3556) the flux density values provided by all the new GMRT observations presented here are in excellent agreement with the spectrum reported in Venturi et al. (1998, 2003), respectively (see Table 6, Sects. 5.2.1 and 5.2.2, Figs. 5-7).

\subsection{Spectral fits}

We fitted the spectra of the central regions of the galaxies in our sample using the Synage++ package (Murgia et al. 2011), to obtain an estimate of the radiative ages of the sources. We found that different radiative loss models were best suited in the various cases (see Table 7). In particular:

- The spectra of J 1254-2900, J 1254-2901a, and J 1254-2904 (i.e. the BCG and the two radio tails in A $3528 \mathrm{~N}$ ), J 12542913 and J 1254-2916 (i.e. the BCG and the tail in A 3528 S), $\mathrm{J} 1257-3021$ (i.e. the BCG in A 3532), and J 1324-3140 (i.e. the BCG in A 3556) are best fitted by a power law;

- J 1324-3138 (i.e. the radio tail in A 3556) is best fitted by a Jaffe \& Perola model (JP; Jaffe \& Perola 1974), which assumes continuous isotropisation of the pitch angle of the radiating electrons by electron scattering, whose timescale is much shorter than their radiative lifetime;

- None of the above models provides an acceptable fit to the spectrum of $\mathrm{J} 1327-3129 \mathrm{~b}$ (i.e. the BCG in A 3558), which we interpret as another piece of evidence in favour of the possibility that this is a compact steep spectrum source. 
Table 7. Best-fit model of the radiative losses of the central region of the radio galaxies and their physical parameters.

\begin{tabular}{|c|c|c|c|c|c|c|c|c|c|}
\hline $\begin{array}{l}\text { Radio } \\
\text { galaxy }\end{array}$ & $\begin{array}{l}\text { Cluster } \\
\text { name }\end{array}$ & Comp. & $\begin{array}{c}\text { Rad. loss } \\
\text { model }\end{array}$ & $\alpha_{\text {inj }}$ & $\chi_{\text {red }}^{2}$ & $\begin{array}{c}v_{\mathrm{br}} \\
(\mathrm{GHz})\end{array}$ & $\begin{array}{c}B_{\mathrm{eq}} \\
(\mu \mathrm{G})\end{array}$ & $\begin{array}{c}P_{\text {int }} \\
\left(\text { dyne } \mathrm{cm}^{-2}\right)\end{array}$ & $\begin{array}{c}t_{\mathrm{rad}} \\
(\mathrm{Myr})\end{array}$ \\
\hline J 1254-2900 & A $3528 \mathrm{~N}$ & Tot. & PL & $0.83_{-011}^{+0.03}$ & 0.11 & $\geq 8.4$ & 10.6 & $6.4 \times 10^{-12}$ & 14 \\
\hline J 1254-2901a & A $3528 \mathrm{~N}$ & Centr. & PL & $0.67^{-0.011}$ & 0.7 & $\geq 8.4$ & 7.2 & $3.1 \times 10^{-12}$ & 22 \\
\hline \multirow{3}{*}{ J 1254-2904 } & \multirow{3}{*}{ A $3528 \mathrm{~N}$} & Centr. & PL & $0.72_{-0.08}^{+0.03}$ & 1.1 & $\geq 8.4$ & 4.7 & $1.2 \times 10^{-12}$ & 34 \\
\hline & & Diff. & PL & $1.45^{+0.05}$ & 0.06 & $\geq 8.4$ & 3.5 & $7.2 \times 10^{-13}$ & 41 \\
\hline & & Tot. & PL & $0.76_{-0.13}^{+0.21}$ & 1.5 & $\geq 8.4$ & 4.1 & $9.6 \times 10^{-13}$ & 38 \\
\hline \multirow{3}{*}{ J $1254-2913$} & \multirow{3}{*}{ A $3528 \mathrm{~S}$} & Centr. & PL & $1.01^{-0.13}+0.03$ & 0.3 & $\geq 8.4$ & 8.1 & $3.8 \times 10^{-12}$ & 19 \\
\hline & & Diff. & PL & $\begin{array}{r}-0.10 \\
1.77_{-0.56}^{+0.65}\end{array}$ & 3.1 & $\geq 8.4$ & 10.0 & $5.7 \times 10-12$ & 15 \\
\hline & & Tot. & PL & $1.15_{-0.19}^{+0.06}$ & 0.6 & $\geq 8.4$ & 10.2 & $6.1 \times 10^{-12}$ & 15 \\
\hline \multirow{3}{*}{ J 1254-2916 } & \multirow{3}{*}{ A $3528 \mathrm{~S}$} & Centr. & $\overline{P L}$ & $0.59_{-0.10}^{+0.19}$ & 1.1 & $\geq 8.4$ & 5.2 & $1.6 \times 10^{-12}$ & 31 \\
\hline & & Diff. & PL & $0.93^{-0.21}$ & 1.7 & $\geq 8.4$ & 3.9 & $8.7 \times 10^{-13}$ & 40 \\
\hline & & Tot & PL & $0.61_{-0.24}^{+0.22}$ & 0.03 & $\geq 8.4$ & 4.3 & $1.1 \times 10^{-12}$ & 36 \\
\hline \multirow{3}{*}{ J 1257-3021 } & \multirow{3}{*}{ A 3532} & Centr. & PL & $0.71_{-0.02}^{+0.24}$ & 7.4 & $\geq 8.4$ & 6.8 & $2.7 \times 10^{-12}$ & 24 \\
\hline & & Diff. & PL & $2.63_{-0.037}^{+0.46}$ & 1.6 & $\geq 8.4$ & 4.9 & $1.4 \times 10^{-12}$ & 33 \\
\hline & & Tot. & PL & $0.72^{+0.013}$ & 13 & $\geq 8.4$ & 6.7 & $2.6 \times 10^{-12}$ & 24 \\
\hline \multirow{2}{*}{ J 1324-3138 } & \multirow{2}{*}{ A 3556} & Centr. & JP & $0.5^{-0.6}$ & 6.7 & $3.9_{-02}^{+0.1}$ & 3.4 & $6.7 \times 10^{-13}$ & 62 \\
\hline & & Diff. & JP & $0.95_{-0.07}^{+0.03}$ & 2.1 & $11.2^{-0.2} .3$ & 2.9 & $4.9 \times 10^{-13}$ & 39 \\
\hline J 1324-3140 & A 3556 & Centr. & PL & $0.57_{-0.11}^{+0.04}$ & 0.7 & $\geq 8.4$ & 5.2 & $1.5 \times 10^{-12}$ & 32 \\
\hline
\end{tabular}

Notes. ${ }^{(a)}$ Fixed $\alpha$ value. For all the PL models we obtain an upper limit estimation of the radiogalaxy lifetime.

The best-fit values for the break frequency $v_{\mathrm{br}}$ are reported in Table 7. For the sources fitted with a power law, the break frequency is a lower limit, and the radiative age is an upper limit.

We used the break frequencies from the spectral fits (see Table 7) to estimate the radiative age of all the radio galaxies in the sample, under the assumption of equipartition (Govoni \& Feretti 2004), by means of the relation

$t_{\mathrm{rad}}=1590\left[(1+z) v_{\mathrm{br}}\right]^{-1 / 2} \frac{B_{\mathrm{eq}}^{1 / 2}}{B_{\mathrm{eq}}^{2}+B_{\mathrm{CMB}}^{2}} \quad(\mathrm{Myr})$

where $B_{\mathrm{CMB}}=3.25(1+z)^{2}$. The magnetic field is expressed in $\mu \mathrm{G}$, and the frequency in $\mathrm{GHz}$. To estimate $B_{\text {eq }}$, that is the magnetic field at the equipartition, we assumed elliptical geometry (i.e. $V=a b \pi / 6$, with $a$ and $b$ the major and minor semi-axes of the ellipse, respectively), a filling factor of unity, and equal energy contribution in relativistic ions and electrons $(k=1)$ to the total particle energy

$B_{\mathrm{eq}}=\sqrt{\frac{24 \pi}{7} \frac{E_{\mathrm{tot}, \mathrm{min}}}{V}}$,

where $E_{\mathrm{tot}, \mathrm{min}} / V=2.5 \times 10^{41}(1+k)^{4 / 7} P_{1.4 \mathrm{GHz}}^{4 / 7} V^{3 / 7}$, and $P_{1.4 \mathrm{GHz}}$ the radio power of the radio galaxy at $1.4 \mathrm{GHz}$, (e.g. Govoni \& Feretti 2004).

Our results are reported in Table 7 . The radio galaxies span a power range of $22<\log P_{1.4 \mathrm{GHz}\left[\mathrm{WHz}^{-1}\right]}<25$. The most powerful objects are associated with the BCGs of the A 3528 complex, while the weakest are associated with the radio galaxies belonging to the A 3558 complex. The equipartition values for the magnetic field and internal pressure are typical of radio galaxies in clusters (e.g. Feretti et al. 1992; Parma et al. 1999). The internal pressure is higher for the most powerful radio galaxies. The radiative ages for the central regions of the sources in our sample are on average of the order of few times $10^{7} \mathrm{yr}$, in agreement with works in the literature (e.g. Murgia et al. 2011).
For the three BCGs J 1254-2913 (A 3528 S), J 1257-3021 (A 3532), and J 1324-3140 (A 3556) our observations show the presence of extended emission with such a steep spectrum which seems difficult to reconcile with the active region. In all cases the steep spectrum radio emission extends well outside the optical counterpart.

\section{The host galaxies}

The optical (VST OmegaCAM $g$ band) images of the host galaxies are shown in Fig. A.1. The visual inspection of the images is sufficient to reveal the early-type nature of all galaxies in the sample. In most of them, the surface brightness declines smoothly from the centre to the outskirts, with no sign of spiral structure or star-formation knots. There are, however, two galaxies showing evidence of interaction, J 1333-3140 (BCG in A 3562) and J 1254-2913 (BCG in A 3528 S).

On the outskirts of J 1333-3140, a "ripple" (or "shell", see Buta (2013)) is clearly visible in the north-west direction at $\sim 25-30$ arcsec (i.e. $\sim 25-30 \mathrm{kpc}$ at galaxy redshift) indicated by the white arrows in the last panel in Fig. A.1 and in Fig. A.2. A faint fan-shaped brightness feature is visible on the opposite side, plus a clear and a low surface brightness area extending $\sim 80$ arcsec to the east. All these features have the same colours and form a continuous brightness distribution with the rest of the galaxy (see Fig. A.2), indicating that they consist of the same stellar populations. Such kinds of features are typical of the last phases of a merger (e.g. Buta 2013; Bienayme et al. 1985, and references therein).

An enlarged view of the central region of J 1254-2913 is shown in the left panel in Fig. 8. A round-shaped, faint secondary maximum of surface brightness is visible about $3 \mathrm{kpc}$ east from the centre of the galaxy. Another faint feature protrudes for about $2 \mathrm{kpc}$ south-south-east from the same centre. To investigate the nature of the secondary maximum, we show in the right panel of Fig. 8 the $g-i$ colour distribution in the inner $8^{\prime \prime} \times 6^{\prime \prime}$ of J 1254-2913. It is apparent that the $g-i$ colour and the smooth 

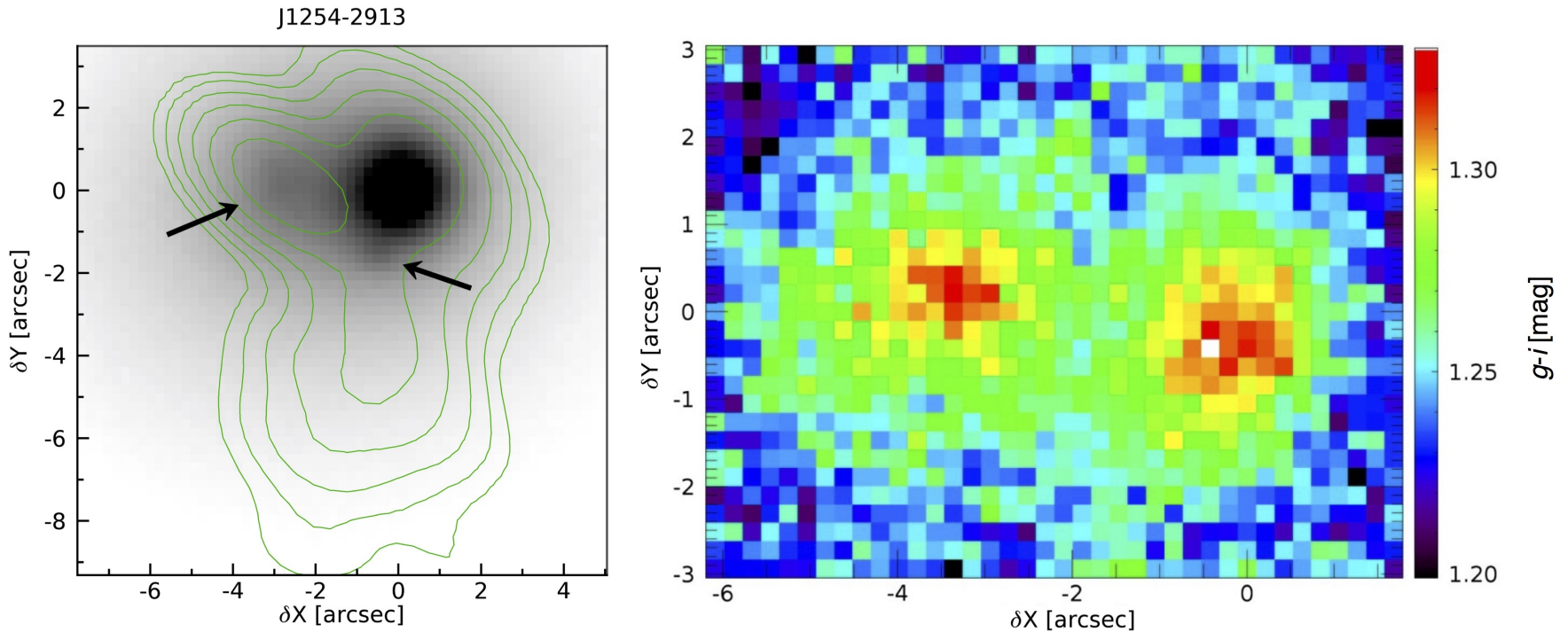

Fig. 8. Left panel: VST-OmegaCAM $i$-band image of the BCG J1254-2913 with the $8.4 \mathrm{GHz}$ radio contours overlaid (green). The left arrow points to a second nucleus, while the right arrow indicates a possible jet. Right panel: $g-i$ color map of the inner $8^{\prime \prime} \times 6^{\prime \prime}$ of J1254-2913. We notice that the $g-i$ colours in the main nucleus of the galaxy (right in the figure) and in the secondary nucleus are similar and have similar gradients.

colour gradient of the secondary maximum are similar to the nucleus of the galaxy, while the range of values $(1.2-1.34 \mathrm{mag})$ is typical of early-type galaxy populations (e.g. Gavazzi et al. 2010). The secondary maximum is therefore the nucleus of an early-type galaxy. The colour of the southern protrusion cannot be measured because of the low signal-to-noise ratio. Both features are accurately traced by the $8.4 \mathrm{GHz}$ VLA contours, suggesting that they could belong to the same galaxy. Due to their low surface brightness, we were not able to measure their redshift, hence the possibility of background or foreground objects, though unlikely, cannot be excluded. The presence of a double nucleus, together with the almost unperturbed overall structure may indicate the final (pre-coalescence) phase of a minor merger (e.g. Gimeno et al. 2004; Lotz et al. 2010; Mezcua et al. 2014), in which the nucleus of the secondary, less massive galaxy is sinking in the host potential well through dynamical friction.

All the BCGs, with the exception of J 1333-3140 (in A 3562), have been observed and classified as $\mathrm{CD}$ galaxies by Fasano et al. (2010). The homogeneous wavelength coverage available for all the galaxies in both complexes comprises the gi optical filters from VST and the WISE channels W1-W3 (at 3.4, 4.6, and $12 \mu \mathrm{m})$. The corresponding magnitudes, corrected for Galactic extinction, are listed in Table 8. The optical magnitudes were obtained with Sextractor (Bertin \& Arnouts 1996). The values in Table 8 are Kron magnitudes, which correspond to about $90 \%$ of the total flux from the galaxy. For extended objects like our galaxies, an accurate derivation of the WISE magnitudes is crucial. The WISE magnitudes for extended objects (gmag in the WISE archive) are measured within the Two Micron All-Sky Survey (2MASS) $K$-band isophototal aperture at $20 \mathrm{mag} \mathrm{arcsec}^{-2}$. There are, however, two 2MASS estimates of the total flux from extended sources, given in the Extended Source Catalogue $\left(k_{\mathrm{m}_{\mathrm{k} 20 \mathrm{fe}}}\right.$ and $\left.k_{\mathrm{m}_{\mathrm{ext}}}\right)$, the second of which is based on extrapolation of the growth curves. These extend out to different radii. We adopted the WISE magnitude measured in the aperture closer to that used for the $i$-band magnitude. If it is the $k_{\mathrm{m}_{\mathrm{ext}}}$ magnitude (curve of growth), then we offset all the WISE magnitudes by the difference in the two 2MASS $K$-band magnitudes $\left(k_{\mathrm{m}_{\mathrm{k} 20 \mathrm{fe}}}-k_{\mathrm{m}_{\mathrm{ext}}}\right)$.
Figure 9, drawn from Jarrett et al. (2017), shows the WISE colours ranges characterizing different galaxy types according to their star-formation or nuclear activity. The colours of all galaxies in our sample are well within the area relevant to the spheroidal passive galaxies, as also indicated by their morphology. We used the fluxes in the wavebands of Table 8 to derive the stellar masses for the galaxies (last column in the table) with the spectral energy distribution (SED) fitting technique using the code MAGPHYS (da Cunha et al. 2008). The code adopts the Bruzual \& Charlot (2003) library of models with a Chabrier (2003) stellar initial mass function and a metallicity value in the range $0.022-1 Z_{\odot}$. The age of the galaxy is free to vary with an upper limit imposed by the age of the Universe at the considered redshift. In order to find the best-fit model, MAGPHYS uses a Bayesian approach. As outputs, it produces the probability distribution function of all the parameters and their best-fit values. For the stellar masses we adopted the median value as the more robust estimate. We run MAGPHYS using a library of templates heavily biased to fit old stellar populations with very little star formation (kindly provided by E. da Cunha), since MAGPHYS, when run on its public library, tends to favour dusty star formation models rather than passive models (e.g. Annunziatella et al. 2014).

We find that the mean stellar masses of the BCGs are $\left\langle\log \left(M_{\star} / M_{\odot}\right)\right\rangle=11.66 \pm 0.09$ in the A 3558 complex and $\left\langle\log \left(M_{\star} / M_{\odot}\right)\right\rangle=11.69 \pm 0.10$ in the A 3528 complex. The mean stellar masses of the tailed galaxies are $\left\langle\log \left(M_{\star} / M_{\odot}\right)\right\rangle=$ $10.09 \pm 0.09$ and $\left\langle\log \left(M_{\star} / M_{\odot}\right)\right\rangle=11.04 \pm 0.24$ in the A 3558 and A 3528 complex, respectively.

\section{Discussion}

We present a detailed radio analysis of the BCGs and tailed radio galaxies in the A 3528 and A 3558 cluster complexes, to investigate whether the large-scale environment is influencing the emission and the cycles of activity of the radio galaxies.

Our study was based on proprietary and literature highsensitivity radio observations, obtained with the GMRT at 235, 325, and $610 \mathrm{MHz}$; Molonglo Observatory Synthesis Telescope (MOST) at $843 \mathrm{MHz}$; VLA at 1.4 and $8.4 \mathrm{GHz}$; ATCA at 
Table 8. Photometric properties and stellar masses of the host galaxies.

\begin{tabular}{|c|c|c|c|c|c|c|c|c|}
\hline Object & $\mathrm{RA}(\mathrm{J} 2000)$ & $\operatorname{Dec}(\mathrm{J} 2000)$ & $g$ & $i$ & W1 & W2 & W3 & $\log \left(M_{\star} / M_{\odot}\right)$ \\
\hline \multicolumn{9}{|l|}{ A $3528 N$} \\
\hline J1254-2900 & 125422.24 & -290046.73 & $4.09 \pm 0.01$ & $2.85 \pm 0.01$ & $10.07 \pm 0.01$ & $10.09 \pm 0.01$ & $9.65 \pm 0.05$ & $11.75 \pm 0.08$ \\
\hline J1254-2901a & 125423.22 & -290103.05 & $15.89 \pm 0.01$ & $1.76 \pm 0.01$ & $12.46 \pm 0.02$ & $12.45 \pm 0.03$ & $11.60 \pm 0.19$ & 0.10 \\
\hline J1254-2901b & 125440.75 & -290148.55 & $16.23 \pm 0.01$ & $15.12 \pm 0.01$ & $12.63 \pm 0.01$ & $12.66 \pm 0.02$ & $12.40 \pm 0.30$ & $10.71 \pm 0.09$ \\
\hline J1254-2904 & 125420.41 & -290408.67 & $15.87 \pm 0.01$ & $14.76 \pm 0.01$ & $12.39 \pm 0.01$ & $12.35 \pm 0.01$ & $12.15 \pm 0.20$ & $10.85 \pm 0.10$ \\
\hline \multicolumn{9}{|l|}{ A $3528 \mathrm{~S}$} \\
\hline J1254-2913 & 125441.10 & -291339.58 & $13.46 \pm 0.01$ & $.33 \pm 0.01$ & $.81 \pm 0.01$ & $9.78 \pm 0$ & $9.44 \pm$ & $11.89 \pm 0.09$ \\
\hline $\begin{array}{l}\mathrm{J} 1254-2916 \\
\text { A } 3532\end{array}$ & 125452.42 & -291616.80 & $14.88 \pm 0.01$ & $13.72 \pm 0.01$ & $11.22 \pm 0.01$ & $11.25 \pm 0.01$ & $10.84 \pm 0.13$ & 11.2 \\
\hline $\begin{array}{l}\mathrm{J} 1257-3021 \\
\text { A } 3556\end{array}$ & 125721.94 & -302149.00 & $14.34 \pm 0.01$ & $13.27 \pm 0.01$ & $10.87 \pm 0.01$ & $10.87 \pm 0.01$ & $10.54 \pm 0.11$ & $11.43 \pm 0.10$ \\
\hline J1324-3138 & 132357.61 & -313844.82 & $14.69 \pm 0.01$ & $13.46 \pm 0.01$ & $10.91 \pm$ & $10.90 \pm 0.01$ & $10.49 \pm$ & $11.38 \pm 0.08$ \\
\hline $\begin{array}{l}\mathrm{J} 1324-3140 \\
\text { A } 3558\end{array}$ & 132406.73 & -314011.56 & $13.89 \pm 0.01$ & $12.74 \pm 0.01$ & $10.01 \pm 0.01$ & $10.00 \pm 0.01$ & $9.62 \pm$ & $11.66 \pm 0.07$ \\
\hline $\begin{array}{l}\mathrm{J} 1327-3129 \mathrm{~b} \\
\text { A } 3562\end{array}$ & 132756.86 & -312945.32 & $13.29 \pm 0.01$ & $12.17 \pm 0.01$ & $9.39 \pm 0.01$ & $9.49 \pm 0.01$ & $9.13 \pm 0.11$ & $11.85 \pm 0.08$ \\
\hline J1333-3140 & 133 & -314 & $14.13 \pm 0$ & $12.97 \pm 0$ & $10.77 \pm$ & $10.81 \pm 0.01$ & $10.34 \pm 0.07$ & $11.46 \pm 0.09$ \\
\hline J1333-3141 & 133331.63 & -314058.33 & $16.30 \pm 0.01$ & $15.09 \pm 0.01$ & $12.63 \pm 0.02$ & $12.80 \pm 0.05$ & $12.57 \pm 0.39$ & $10.70 \pm 0.08$ \\
\hline
\end{tabular}

Notes. Columns 2 and 3: galaxy coordinates corresponding to the photometric centre in $g$ band. Columns 4 and 5: optical ( $g i$ ) magnitudes from VST given in AB photometric system (see Merluzzi et al. 2015; Mercurio et al. 2015). Columns 6-8: mid-infrared (mid-IR) magnitudes from WISE channels W1, W2 and W3 derived as explained in the text. All magnitudes are corrected for the Galactic extinction following Schlafly \& Finkbeiner (2011). Column 9: logarithm of stellar mass in units of solar mass.

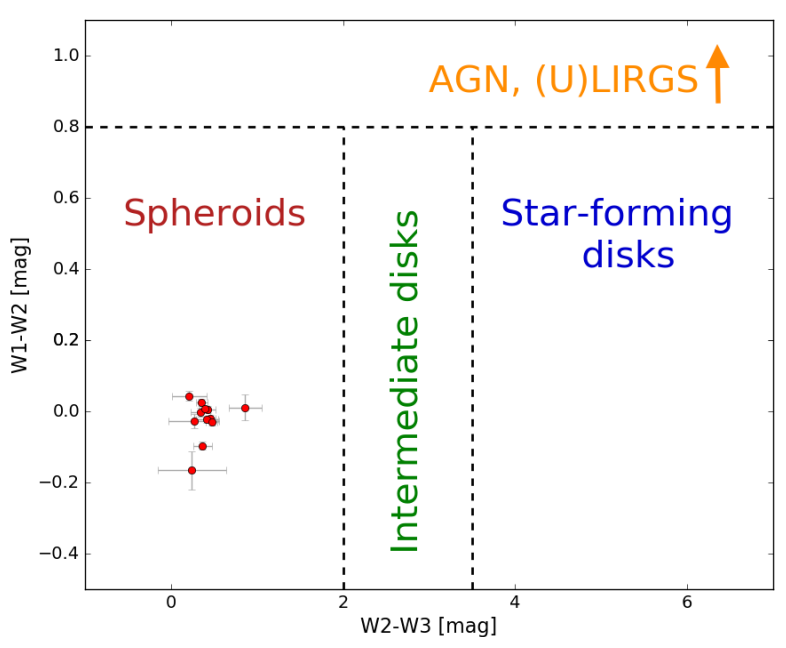

Fig. 9. Position of the 12 radio galaxies (dots with error bars) in the WISE colour-colour diagram. The diagram is divided into regions of colours characterizing different galaxy types following Jarrett et al. (2017; cf. their Fig. 11).

1.38, 2.38 and $5 \mathrm{GHz}$. The multi-frequency radio images show that the radio properties of the brightest cluster galaxies in the A 3528 and A 3558 complex are very different, as detailed below.

\subsection{The BCGs}

The two BCGs located at the centre of the main clusters in the two complexes, J 1254-2900 in A $3528 \mathrm{~N}$ and J 1327-3129b in A 3558, are both embedded in the faint optical halo emission, but their radio properties are completely different. The former has a clear mini-double morphology (linear size of about
$30 \mathrm{kpc}$ ) and its spectrum is well modelled by a power law, with a spectral index typical of an active source $(\alpha \approx 0.8)$, implying a young population of relativistic electrons. Moreover, it is powerful in the radio band $\left(\log P_{1.4 \mathrm{GHz}\left[\mathrm{W} \mathrm{Hz}^{-1}\right]} \approx 24\right)$. On the contrary, $\mathrm{J} 1327-3129 \mathrm{~b}$ (A 3558) is compact, small (linear size of $\approx 20 \mathrm{kpc}$ ) and very weak $\left(\log P_{1.4 \mathrm{GHz}\left[\mathrm{W} \mathrm{Hz}^{-1}\right]} \approx 22.5\right)$. Its spectrum is concave and is suggestive of a compact steep-spectrum (CSS) radio galaxy, that is a radio galaxy in the very early stages of its evolution. The very steep spectrum in the optically thin part of the spectrum further suggests the presence of a population of old electrons. Short Chandra observations (ObsID 1646) have also revealed the presence of a cool corona in the $1-2 \mathrm{keV}$ band surrounding the optical and radio emission (Sun 2009). Overall, the radio and X-ray properties of $\mathrm{J} 1327-3129 \mathrm{~b}$ suggest that the BCG in A 3558 is an old CSS, whose radio emission is completely confined by the X-ray corona. The study of the interplay between the radio emission of the galaxy and the ICM will be presented in a future work.

We postulate that the differences of the radio properties of these two BCGs are related to the different environment in which they are located. Previous studies of the A 3528 complex (Gastaldello et al. 2003) suggest that the individual clusters have not crossed each other, and their nuclear regions have not yet undergone major disruption. This is confirmed by the presence of cool-cores (see Fig. 7 in Gastaldello et al. 2003), an indication that it is a relaxed system. On the other hand, multi-band observations provide support to the idea that the A 3558 complex has undergone a major merger, particularly active in the region between A 3562 and A3558 (e.g. Bardelli et al. 1998a,b; Merluzzi et al. 2016; Ettori et al. 2000; Rossetti et al. 2007; Ghizzardi et al. 2010; Venturi et al. 2000, 2003, 2017; Giacintucci et al. 2004, 2005). Considering that the BCG in A 3562 is radio quiet, and the well-known merging state from studies of the literature, our result is another piece of evidence suggesting that another observational implication of 
major mergers is the quenching of the nuclear radio emission in BCGs (Kale et al. 2015). Despite that, we are aware of the fact that the interplay between the cluster dynamics and the radio emission properties of cluster galaxies is not fully understood yet, and that a number of BCGs are also found radio quiet in relaxed environments (Sun 2009).

The remaining BCGs in our sample, J 1254-2913 (A 3528 S), $\mathbf{J}$ 1257-3021 (A 3532) and $\mathbf{J} 1324-3140$ (A 3556), are similar in their global properties. They are all powerful in the radio band $\left(\log P_{1.4 \mathrm{GHz}\left[\mathrm{W} \mathrm{Hz}^{-1}\right]} \approx 23-25\right)$. They are characterised by a compact active region and by diffuse emission, which extends well beyond the optical counterpart, and has a radio spectrum much steeper than the nuclear component. Their overall morphology, the clear separation between the active nucleus and the diffuse emission, and the much steeper spectrum of the latter suggest that these objects could be examples of restarted radio sources.

We point out that a number of restarted radio galaxies have been found at the centres of relaxed groups and clusters (e.g. Giacintucci et al. 2007, 2014b; Murgia et al. 2011; Shulevski et al. 2015), as it is indeed the case of A 3528 S, A 3532 and A 3556. Indeed the latter does not seem to be involved in the major merging occurring in the supercluster core (see above).

We do not observe such differences in the optical properties of the host galaxies of the BCGs in the two cluster complexes (Fig. 9). They are all passive, belong to the red-sequence, do not show relevant signs of (un)obscured star formation, and have on average the same high stellar masses $\left(\left\langle\log \left(M_{\star} / M_{\odot}\right)\right\rangle=11.33\right)$. We speculate that cluster mergers do not affect the host galaxies, at least not on the life timescale typical of the radio emission, that is of the order of $10^{7}-10^{8}$ years (i.e $1.4-7.8 \times 10^{7}$ years; see Murgia 2003; Murgia et al. 2011).

\subsection{Tails and cluster weather in $A 3528$}

In contrast to the A 3558 complex, where there is substantial observational support in favour of merger and accretion activity, little is known about the A 3528 complex, in particular concerning the two sub-clusters A $3528 \mathrm{~N}$ and A $3528 \mathrm{~S}$. Studies in the optical band (Bardelli et al. 2001) show that the galaxy velocity distribution is characterised by the presence of several peaks (see Fig. 4a in Bardelli et al. 2001), which is suggestive of substructure, and possibly indicating some sort of interaction between the two sub-clusters. Despite that, Bardelli et al. also found that this velocity distribution is well approximated by a Gaussian, which implies that any interaction between these two sub-clusters, if indeed present, has not affected the observed radio and optical properties of the galaxy population yet. Following this interpretation, the thermal properties of the ICM (Gastaldello et al. 2003) show a significant excess in the XMM-Newton surface brightness north-west of A $3528 \mathrm{~N}$ and north-east of A $3528 \mathrm{~S}$. This information has been interpreted as a scenario in which the two systems are orbiting around each other, as a consequence of an off-axis post-(minor)merger event.

The presence of several tailed radio galaxies in the A 3528 complex is a valuable addition to the available information on the so-far unclear dynamical state of the A 3528 complex. Three tails, J 1254-2901a and J 1254-2901b in A 3528 N and J 12542916 in A $3528 \mathrm{~S}$, all suggest an eastward motion, at least on the plane of the sky. The S-shaped morphology of the small double radio galaxy associated with the BCG in A 3528 is a further suggestion of some form of dynamical activity in this cluster (Gopal-Krishna et al. 2012).
The tailed radio galaxy $\mathrm{J} 1254-2904$ in A $3528 \mathrm{~N}$ is intriguing: it is located between A $3528 \mathrm{~N}$ and A $3528 \mathrm{~S}$, suggesting a galaxy motion northwards, and presents a peculiar bottle-neck morphology possibly due to integration with the ICM. On the basis of XMM-Newton observations, Gastaldello et al. (2003) proposed indeed a scenario in which A $3528 \mathrm{~N}$ and A $3528 \mathrm{~S}$ are moving along the north-west/southeast and north-east/south-west direction, respectively, with a merger occurring about 1-2 Gyr ago that was sufficient to disturb only the outer galaxy distribution but not the cluster cores. Recent numerical simulations (Jones et al. 2017) and new observational results (e.g. Cuciti et al. 2018; de Gasperin et al. 2017; Di Gennaro et al. 2018) have shown that if the jet plasma is impacted perpendicularly by wind (i.e. "cross wind") resulting from a crossing shock, it could be displaced and revived, resulting into the broken-shape morphology we see for this source. Although spectral index analysis across the tail is necessary to confirm such scenario, no clear emission from the tail of the radio galaxy is provided at high frequencies $(>610 \mathrm{MHz})$. Another less exciting possibility is that the southern region of emission is actually associated with the galaxy at $z \sim 0.07$ shown in Fig. 4. Future analysis will be provided with our new upcoming uGMRT observations at $1.4 \mathrm{GHz}$.

The optical analysis of the host galaxies of the tailed radio sources does not show any difference, either between the two cluster complexes and with the BCGs discussed above.

\section{Conclusions}

Our study of the radio properties of the BGCs and tailed radio galaxies in the central region of the Shapley Concentration provides information on different aspects of galaxy clusters and their formation. While the radio properties of the BCGs provide information on the activity state in the central dense environment of the galaxy clusters, tailed radio galaxies are tracers of the motion of the host galaxies within the clusters, which take into account also the bulk flow motion in the cluster weather. The main conclusions of this paper can be summarised as follow:

- Our 235, 325, and $610 \mathrm{MHz}$ radio images have shown different radio galaxy morphology contents for the two cluster complexes: while the A 3558 one is dominated by compact radio sources (Fig. 2), the A 3528 one shows the presence of several radio tails and diffuse radio emission surrounding the BCGs (i.e. J 1254-2913 in A $3528 \mathrm{~S}$ and J 1257-3021 in A 3532, Fig. 1).

- We found powerful radio active BGCs in the A 3528 complex. Two of them (J 1254-2913 and J 1257-3021, located in A 3528 S and A 3532 respectively) are suggestive of restarted activity, with an active nucleus (as clearly derived from its spectral properties) and radio emission with very steep spectrum extending out into the ICM well beyond the boundaries of the optical counterpart and possibly tracing a previous cycle of activity. Interestingly, the broad band properties of these clusters suggest that they are either relaxed, or have not undergone a disruptive merger event.

- The BCGs in A 3558 (i.e. J 1327-3129b) and in A 3562 (i.e. $\mathrm{J} 1333-3141$ ) show remarkable differences from the ones in the A 3528 complex. The former is a faint compact source with concave spectrum, typical of compact steep spectrum sources. The slope in the optically thin part of the spectrum is quite steep, suggesting that this source has already aged and will not evolve in an extended source, that is, that the radio plasma may not expand beyond its present size and the radio source may evolve confined by the external medium. The latter is 
radio quiet at the sensitivity limits of our observations. Despite such differences, considering the several pieces of observational evidence in favour of the fact that A 3558 and A 3562 have undergone a recent major merger event, it is tempting to conclude that this has severely affected the radio emission of the BCGs.

- The BCG in A 3556 (i.e. J 1324-3138), though less powerful, exhibits properties comparable with those of the A 3528 complex, with hints of restarted activity; it has a compact core whose flat spectrum is suggestive of variability, and two faint lobes that are well imaged only at $325 \mathrm{MHz}$ and below. A 3556 is at the extreme western periphery of the A 3558 complex and the available multi-band information suggests that is has not taken part in the major merger event of the A 3558-A 3562 region.

- We performed a study on the host galaxies in both complexes. Not surprisingly, they are associated with high stellar mass passive galaxies. We do not observe any difference between the A 3528 and A 3558 complexes. We speculate that the cluster dynamical state does not affect the optical counterparts of the radio galaxies, at least on the lifetime of the radio emission, $10^{7}-10^{8} \mathrm{yr}$.

A detailed study of the interplay between the diffuse radio emission from the BCGs and the X-ray emission from the ICM, aimed to confirm the hypothesis of restarted radio activity and the presence of X-ray cavities or coronae, is in progress and will be presented in a future work. In particular, we will make use of our new uGMRT observations.

Acknowledgements. We thank the anonymous referee for carefully reading the paper, and for the useful comments and suggestions that have improved the clarity of the manuscript and the presentation of our results. This paper is based on data obtained with the Giant Metrewave Radio Telescope (GMRT) and the Very Large Array (VLA). GMRT is run by the National Centre for Radio Astrophysics of the Tata Institute of Fundamental Research. The National Radio Astronomy Observatory is a facility of the National Science Foundation operated under cooperative agreement by Associated Universities, Inc. The VLA is operated by the National Radio Astronomy Observatory, which is a facility of the National Science Foundation operated under cooperative agreement by Associated Universities, Inc. This research has made use of the NASA/IPAC Extragalactic Database (NED) which is operated by the Jet Propulsion Laboratory, California Institute of Technology, under contract with the National Aeronautics and Space Administration. Basic research in radio astronomy at the Naval Research Laboratory is supported by 6.1 Base funding. CPH acknowledges financial support from PRIN INAF 2014. PM, GB, and AM acknowledge funding from the INAF PRIN-SKA 2017 programme 1.05.01.88.04. The authors thank E. Iodice who collected the $g$-band imaging of the A 3528 complex at VST.

\section{References}

Annunziatella, M., Biviano, A., Mercurio, A., et al. 2014, A\&A, 571, A80 Baars, J. W. M., Genzel, R., Pauliny-Toth, I. I. K., \& Witzel, A. 1977, A\&A, 61, 99

Bardelli, S., Pisani, A., Ramella, M., Zucca, E., \& Zamorani, G. 1998a, MNRAS, 300, 589

Bardelli, S., Zucca, E., Zamorani, G., Vettolani, G., \& Scaramella, R. 1998b, MNRAS, 296, 599

Bardelli, S., Zucca, E., \& Baldi, A. 2001, MNRAS, 320, 387

Barnes, J. E., \& Hernquist, L. E. 1991, ApJ, 370, L65

Bekki, K. 2001, ApJ, 546, 189

Bertin, E., \& Arnouts, S. 1996, A\&AS, 117, 393

Best, P. N., von der Linden, A., Kauffmann, G., Heckman, T. M., \& Kaiser, C. R. 2007, MNRAS, 379, 894

Bienayme, O., Bosma, A., \& Athanassoula, E. 1985, in Cosmical Gas Dynamics, ed. F. D. Kahn, 205

Brunetti, G., \& Jones, T. W. 2014, Int. J. Mod. Phys. D, 23, 1430007

Bruzual, G., \& Charlot, S. 2003, MNRAS, 344, 1000

Burns, J. O. 1998, Science, 280, 400

Buta, R. J. 2013, in Galaxy Morphology, eds. T. D. Oswalt, \& W. C. Keel, 1

Butcher, H., \& Oemler, Jr., A. 1984, ApJ, 285, 426
Byrd, G., \& Valtonen, M. 1990, ApJ, 350, 89

Cassano, R., Ettori, S., Brunetti, G., et al. 2013, ApJ, 777, 141

Cavagnolo, K. W., Donahue, M., Voit, G. M., \& Sun, M. 2008, ApJ, 683, L107

Chabrier, G. 2003, ApJ, 586, L133

Chandra, P., Ray, A., \& Bhatnagar, S. 2004, ApJ, 612, 974

Coppejans, R., van Velzen, S., Intema, H. T., et al. 2017, MNRAS, 467, 2039

Cowie, L. L., \& Songalia, A. 1977, Nature, 266, 501

Cuciti, V., Brunetti, G., van Weeren, R., et al. 2018, A\&A, 609, A61

da Cunha, E., Charlot, S., \& Elbaz, D. 2008, MNRAS, 388, 1595

Dallacasa, D., \& Orienti, M. 2016, Astron. Nachr., 337, 120

de Filippis, E., Schindler, S., \& Erben, T. 2005, A\&A, 444, 387

de Gasperin, F., Intema, H. T., Shimwell, T. W., et al. 2017, Sci. Adv., 3, e1701634

Di Gennaro, G., van Weeren, R. J., Hoeft, M., et al. 2018, ApJ, submitted

Dressler, A. 1980, ApJ, 236, 351

Dressler, A., Oemler, Jr., A., Couch, W. J., et al. 1997, ApJ, 490, 577

Ettori, S., Bardelli, S., De Grandi, S., et al. 2000, MNRAS, 318, 239

Fanaroff, B. L., \& Riley, J. M. 1974, MNRAS, 167, 31P

Fanti, C., Fanti, R., Dallacasa, D., et al. 1995, A\&A, 302, 317

Fasano, G., Bettoni, D., Ascaso, B., et al. 2010, MNRAS, 404, 1490

Feretti, L., Perola, G. C., \& Fanti, R. 1992, A\&A, 265, 9

Feretti, L., \& Venturi, T. 2002, in Merging Processes in Galaxy Clusters, eds. L.

Feretti, I. M. Gioia, \& G. Giovannini, Astrophys. Space Sci. Libr., 272, 163 Gastaldello, F., Ettori, S., Molendi, S., et al. 2003, A\&A, 411, 21

Gavazzi, G., Fumagalli, M., Cucciati, O., \& Boselli, A. 2010, A\&A, 517, A73

Ghizzardi, S., Rossetti, M., \& Molendi, S. 2010, A\&A, 516, A32

Giacintucci, S., Venturi, T., Bardelli, S., Dallacasa, D., \& Zucca, E. 2004, A\&A, 419, 71

Giacintucci, S., Venturi, T., Brunetti, G., et al. 2005, A\&A, 440, 867

Giacintucci, S., Venturi, T., Murgia, M., et al. 2007, A\&A, 476, 99

Giacintucci, S., Markevitch, M., Brunetti, G., et al. 2014a, ApJ, 795, 73

Giacintucci, S., Markevitch, M., Venturi, T., et al. 2014b, ApJ, 781, 9

Giacintucci, S., Markevitch, M., Cassano, R., et al. 2017, ApJ, 841, 71

Gimeno, G. N., Díaz, R. J., \& Carranza, G. J. 2004, AJ, 128, 62

Gopal-Krishna, Biermann, P. L., Gergely, L., \& Wiita, P. J. 2012, Res. Astron. Astrophys., 12, 127

Govoni, F., \& Feretti, L. 2004, Int. J. Mod. Phys. D, 13, 1549

Gregorini, L., de Ruiter, H. R., Parma, P., et al. 1994, A\&AS, 106, 1

Gunn, J. E., \& Gott, III., J. R. 1972, ApJ, 176, 1

Intema, H. T., Jagannathan, P., Mooley, K. P.,\& Frail, D. A. 2017, A\&A, 598, A78

Jaffe, W., \& Perola, G. C. 1974, A\&A, 31, 223

Jarrett, T. H., Cluver, M. E., Magoulas, C., et al. 2017, ApJ, 836, 182

Jones, T. W., Nolting, C., O’Neill, B. J., \& Mendygral, P. J. 2017, Phys. Plasmas, 24, 041402

Kale, R., Venturi, T., Cassano, R., et al. 2015, A\&A, 581, A23

Kauffmann, G., White, S. D. M., Heckman, T. M., et al. 2004, MNRAS, 353, 713

Lakhchaura, K., Singh, K. P., Saikia, D. J., \& Hunstead, R. W. 2013, ApJ, 767, 91

Larson, R. B., Tinsley, B. M., \& Caldwell, C. M. 1980, ApJ, 237, 692

Lewis, I., Balogh, M., De Propris, R., et al. 2002, MNRAS, 334, 673

Liu, W., Sun, M., Nulsen, P., et al. 2018, MNRAS, submitted [arXiv:1806.04692]

Lotz, J. M., Jonsson, P., Cox, T. J., \& Primack, J. R. 2010, MNRAS, 404, 575

Markevitch, M., \& Vikhlinin, A. 1997, ApJ, 474, 84

Markevitch, M., \& Vikhlinin, A. 2007, Phys. Rep., 443,

McNamara, B. R., \& Nulsen, P. E. J. 2007, ARA\&A, 45, 117

McNamara, B. R., \& Nulsen, P. E. J. 2012, New J. Phys., 14, 055023

Mercurio, A., Merluzzi, P., Busarello, G., et al. 2015, MNRAS, 453, 3685

Merluzzi, P., Busarello, G., Haines, C. P., et al. 2015, MNRAS, 446, 803

Merluzzi, P., Busarello, G., Dopita, M. A., et al. 2016, MNRAS, 460, 3345

Mezcua, M., Lobanov, A. P., Mediavilla, E., \& Karouzos, M. 2014, ApJ, 784, 16

Miley, G. 1980, ARA\&A, 18, 165

Moore, B., Katz, N., Lake, G., Dressler, A., \& Oemler, A. 1996, Nature, 379, 613

Murgia, M. 2003, PASA, 20, 19

Murgia, M., Parma, P., Mack, K.-H., et al. 2011, A\&A, 526, A148

Nulsen, P. E. J. 1982, MNRAS, 192, 1007

O’Dea, C. P., \& Owen, F. N. 1985, AJ, 90, 954

Parma, P., Murgia, M., Morganti, R., et al. 1999, A\&A, 344, 7

Pizzo, R. F., \& de Bruyn, A. G. 2009, A\&A, 507, 639

Prasad, J., \& Chengalur, J. 2012, Exp. Astron., 33, 157

Quintana, H., Ramirez, A., Melnick, J., Raychaudhury, S., \& Slezak, E. 1995, AJ, 110, 463

Quintana, H., Melnick, J., Proust, D., \& Infante, L. 1997, A\&AS, 125, 247

Raychaudhury, S. 1989, Nature, 342, 251 
G. Di Gennaro et al.: Cosmic dance in the Shapley Concentration Core. I.

Reid, A. D., Hunstead, R. W., \& Pierre, M. M. 1998, MNRAS, 296, 531

Reisenegger, A., Quintana, H., Carrasco, E. R., \& Maze, J. 2000, AJ, 120, 523

Rossetti, M., Ghizzardi, S., Molendi, S., \& Finoguenov, A. 2007, A\&A, 463, 839

Scaramella, R., Baiesi-Pillastrini, G., Chincarini, G., Vettolani, G., \& Zamorani, G. 1989, Nature, 338, 562

Schindler, S. 1996, MNRAS, 280, 309

Schlafly, E. F., \& Finkbeiner, D. P. 2011, ApJ, 737, 103

Shapley, H. 1930, Harvard Coll. Observat. Bull., 874, 9

Shimwell, T. W., Luckin, J., Brüggen, M., et al. 2016, MNRAS, 459, 277

Shulevski, A., Morganti, R., Barthel, P. D., et al. 2015, A\&A, 579, A27

Stroe, A., van Weeren, R. J., Intema, H. T., et al. 2013, A\&A, 555, A110

Sun, M. 2009, ApJ, 704, 1586

Toomre, A., \& Toomre, J. 1972, ApJ, 178, 623

van Weeren, R. J., Brüggen, M., Röttgering, H. J. A., et al. 2011, A\&A, 533, A35
Venturi, T., Bardelli, S., Morganti, R., \& Hunstead, R. W. 1997, MNRAS, 285 898

Venturi, T., Bardelli, S., Morganti, R., \& Hunstead, R. W. 1998, MNRAS, 298, 1113

Venturi, T., Bardelli, S., Morganti, R., \& Hunstead, R. W. 2000, MNRAS, 314, 594

Venturi, T., Bardelli, S., Zambelli, G., Morganti, R., \& Hunstead, R. W. 2001, MNRAS, 324, 1131

Venturi, T., Bardelli, S., Dallacasa, D., et al. 2003, A\&A, 402, 913

Venturi, T., Giacintucci, S., Dallacasa, D., et al. 2008, A\&A, 484, 327

Venturi, T., Bardelli, S., Dallacasa, D., et al. 2017, Galaxies, 5, 16

Vettolani, G., Chincarini, G., Scaramella, R., \& Zamorani, G. 1990, AJ, 99, 1709

Wright, E. L., Eisenhardt, P. R. M., Mainzer, A. K., et al. 2010, AJ, 140, 1868

Zucca, E., Zamorani, G., Scaramella, R., \& Vettolani, G. 1993, ApJ, 407, 470

ZuHone, J. A., Markevitch, M., Brunetti, G., \& Giacintucci, S. 2013, ApJ, 762, 


\section{Appendix A: Optical images of the host galaxies}

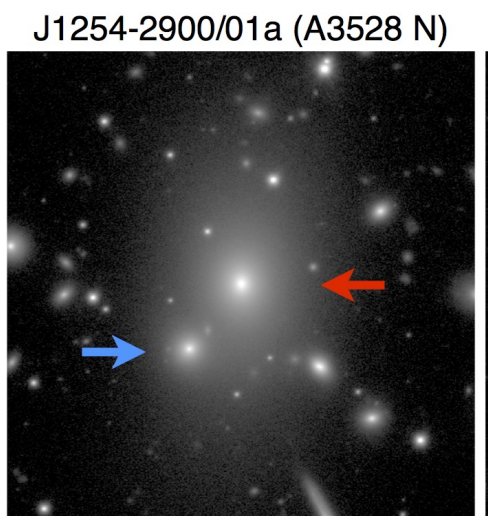

J1254-2913 A3528 S)

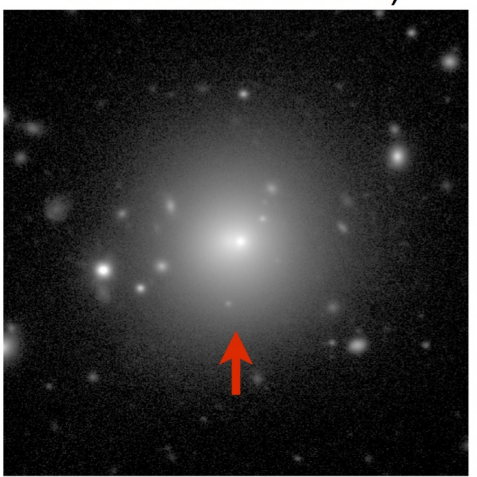

J1324-3138 (A3556)

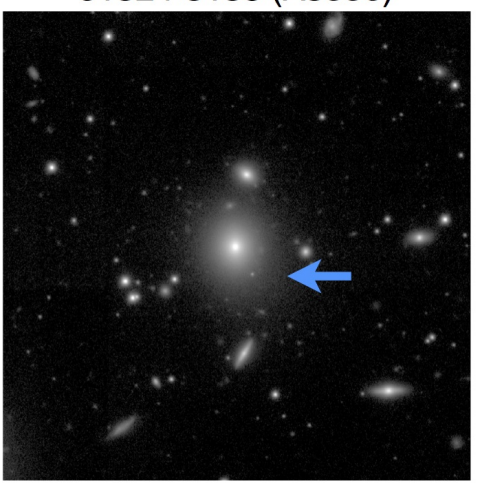

J1327-3129b (A3558)

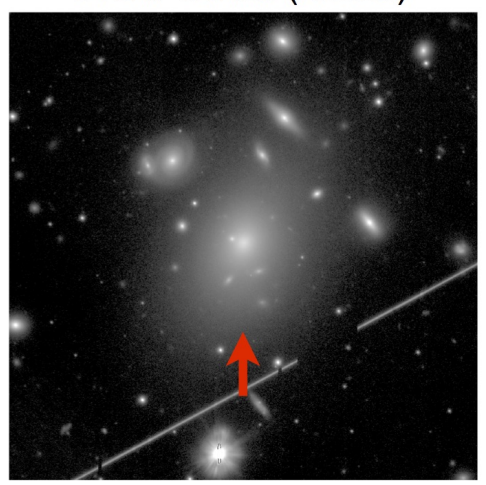

J1254-2901b A3528 N)

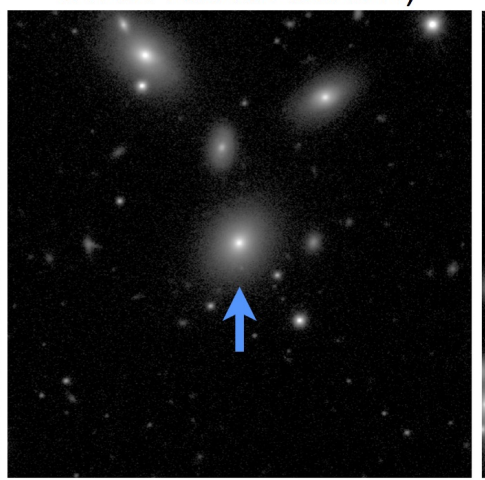

J1254-2916 A3528 S)

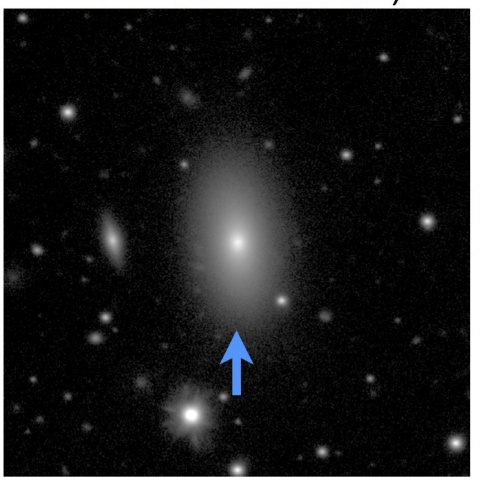

J1324-3140 (A3556)

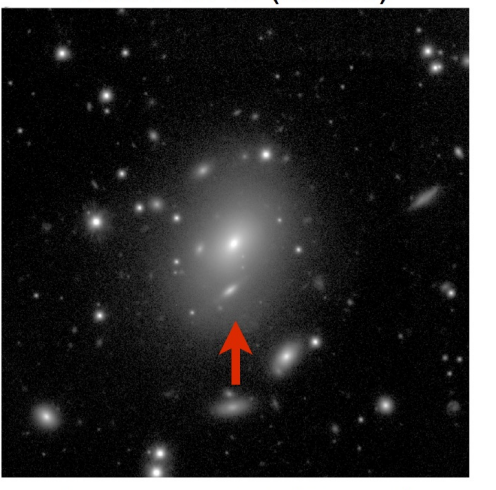

J1333-3140/41 (A3562)

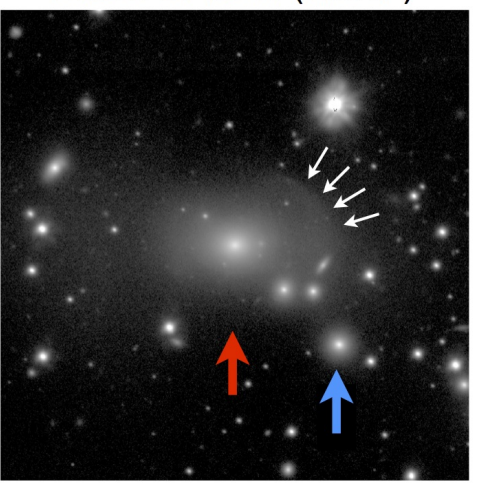

J1254-2904 A3528 N)

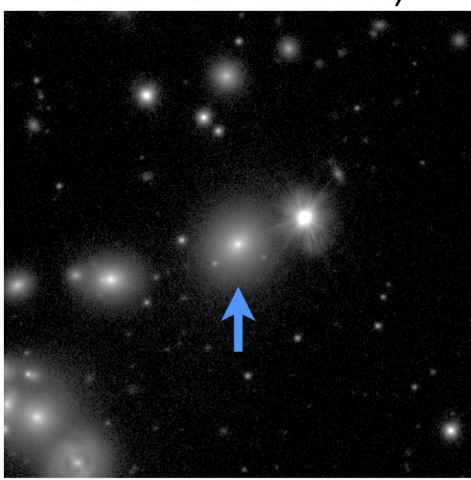

J1257-3021 A3532)

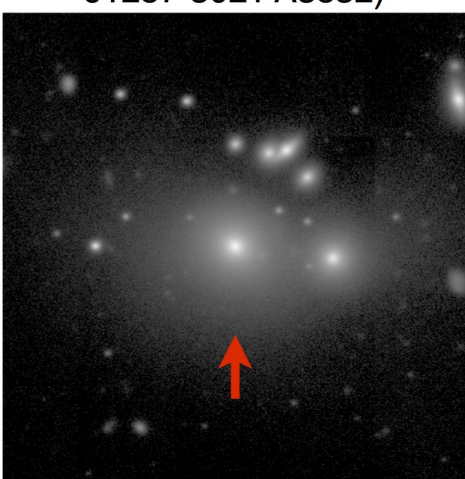

$$
\text { (1) }
$$


G. Di Gennaro et al.: Cosmic dance in the Shapley Concentration Core. I.

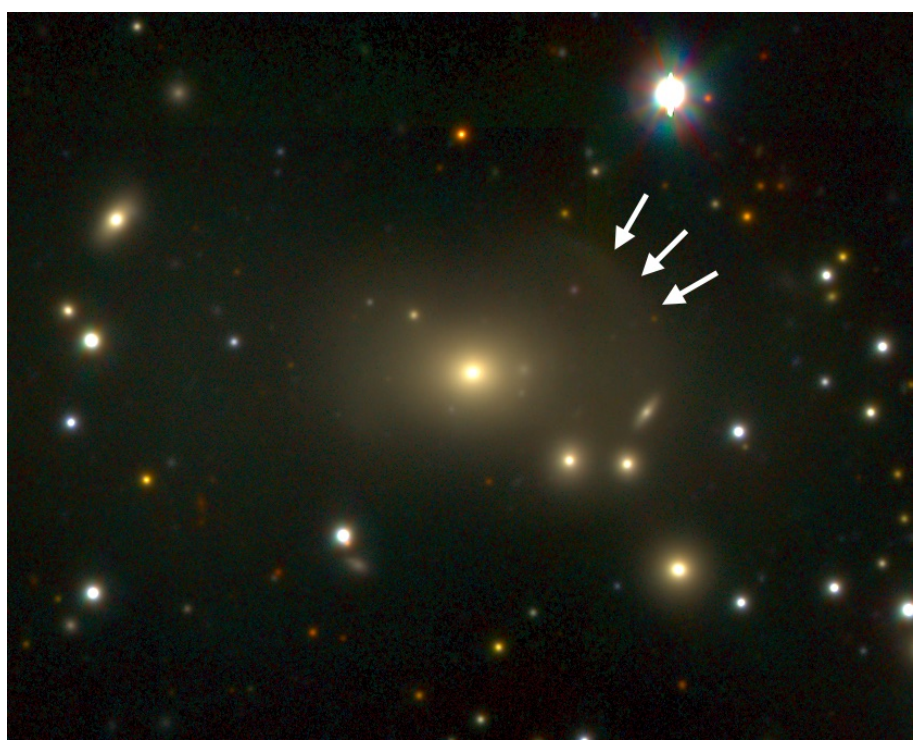

Fig. A.2. VST-ACESS gri composite images of the BCG in A 3562 which shows similar brightness distribution from the shell towards the optical cD position. As for the last panel in Fig. A.1, the white arrows highlight the position of the optical shell. 